\title{
A LOGIC FOR METRIC AND TOPOLOGY
}

\author{
FRANK WOLTER AND MICHAEL ZAKHARYASCHEV
}

\begin{abstract}
We propose a logic for reasoning about metric spaces with the induced topologies. It combines the 'qualitative' interior and closure operators with 'quantitative' operators 'somewhere in the sphere of radius $r$,' including or excluding the boundary. We supply the logic with both the intended metric space semantics and a natural relational semantics, and show that the latter (i) provides finite partial representations of (in general) infinite metric models and (ii) reduces the standard ' $\varepsilon$-definitions' of closure and interior to simple constraints on relations. These features of the relational semantics suggest a finite axiomatisation of the logic and provide means to prove its EXPTIME-completeness (even if the rational numerical parameters are coded in binary). An extension with metric variables satisfying linear rational (in)equalities is proved to be decidable as well. Our logic can be regarded as a 'well-behaved' common denominator of logical systems constructed in temporal, spatial, and similarity-based quantitative and qualitative representation and reasoning. Interpreted on the real line (with its Euclidean metric), it is a natural fragment of decidable temporal logics for specification and verification of real-time systems. On the real plane, it is closely related to quantitative and qualitative formalisms for spatial representation and reasoning, but this time the logic becomes undecidable.
\end{abstract}

$\S 1$. Introduction. The concept of a metric space with the induced topology is obviously fundamental for any serious course in mathematics or computer science. Standard exercises an undergraduate student is confronted with look as follows. Let $\langle V, d\rangle$ be a metric space, $X \subseteq V$ and $a>0$.

(1) Is the set $A=\{y \in V \mid \exists x \in X d(x, y)<a\}$ open?

(2) If $y \in V$ belongs to the topological closure of the set $A$ above, does it mean that there exists $x \in X$ such that $d(y, x) \leq a$ ?

A proof of (1) usually involves an arbitrarily small $\varepsilon>0$. A counterexample for (2) can only be found in the class of infinite metric spaces.

The main aim of this paper is to find out to which extent this kind of reasoning can be mechanised - e.g., is it decidable? what is its computational complexity? and whether reasoning with the 'arbitrarily small $\varepsilon>0$ ' and infinite spaces can be performed using finitely many logical rules and finite models. In this perspective, our direction of research can be regarded as a 'metric extension' of Tarski's programme of the algebraisation of topology ("of creating an algebraic apparatus adequate for the treatment of portions of point-set topology," to be more precise) [29]; see also [34, 25, 6, 30, 1]. 
There is another idea underpinning this paper. As is well-known, reasoning about metric and topology is fundamental in various areas of computer science. For example:

- Temporal logics for specification and verification of real-time systems deal with the real line $\mathbb{R}$ and the standard Euclidean metric (see, e.g., [4, 23]). Using their qualitative temporal operators such as 'since' and 'until' interpreted by the metric of $\mathbb{R}$, one can define the topological closure and interior over $\mathbb{R}$. For example, a point $x$ is in the topological closure of a set $X$ if and only if there is no point $y>x$ until which 'not $X$ ' and there is no point $y<x$ since which 'not $X$.'

- Spatial representation and reasoning uses various topological and metric relations between regions $[29,16,21,7,31,1]$. The intended models are based on - among others and in the decreasing order of abstractnessarbitrary topological spaces, metric spaces with their topologies, and the two-dimensional Euclidean space $\mathbb{R}^{2}$ (see, e.g., [36, 14]).

- Similarity measures that are used to classify various sets of objects (e.g., proteins or viruses in bio-informatics) give rise to reasoning about metric spaces not related at all to the standard Euclidean spaces [13]. [18, 19] suggest a combination of topological relations between regions in spaces with similarity measures for classifying and identifying objects. Logics for reasoning about similarity have been developed in the field of approximate reasoning $[15,17]$.

In this respect, we are looking for a logic which can be regarded as a sort of 'common denominator' of the formalisms constructed in these fields and which reveals most important expressivity and complexity issues that arise in special purpose temporal, spatial, and similarity logics. (In modal logic, such a position is occupied by the minimal logic $\mathbf{K}$ which is a 'common denominator' of various 'qualitative' temporal, description, spatial, epistemic, dynamic, etc. logics.)

The logic we construct here is a natural combination of the logic of metric spaces from [35] which comes equipped with the operators

- $\exists^{<a}$ for 'somewhere in the sphere of radius $a$ excluding its boundary,' where $a \in \mathbb{Q}^{+}$,

- $\exists \leq a$ for 'somewhere in the sphere of radius $a$ including the boundary,' where $a \in \mathbb{Q}^{+}$,

and the well established logic $\mathbf{S} \mathbf{4}_{u}$ of topological spaces which has the operators of the standard modal logic $\mathbf{S} 4$, namely,

- $\square$ for topological interior and

- $\diamond$ for topological closure,

as well as the 'universal modalities'

- $\forall$ for 'everywhere in the space' and

- $\exists$ for 'somewhere in the space.'

Besides the intended metric space models, we supply the logic with a natural relational semantics and show that it (i) provides finite partial representations of (in general) infinite metric models and (ii) reduces the standard ' $\varepsilon$-definitions' of closure and interior to simple constraints on relations. Using these features of 
the relational semantics, we give a finite axiomatisation of the logic and prove its EXPTIME-completeness (even if the numerical parameters are coded in binary). An extension with metric variables satisfying linear rational (in)equalities is proved to be decidable as well.

\section{$\S 2$. Semantics and syntax.}

Intended models. A metric model is a structure of the form

$$
\mathfrak{M}=\left\langle V, d, P_{1}^{\mathfrak{M}}, P_{2}^{\mathfrak{M}}, \ldots\right\rangle,
$$

where the $P_{i}^{\mathfrak{M}}$ are subsets of a nonempty set $V$ and $d$ is a function from $V \times V$ into the set $\mathbb{R}^{+}$of non-negative real numbers such that $\langle V, d\rangle$ is a metric spacei.e., it satisfies the standard metric axioms

$$
\begin{aligned}
& d(x, y)=0 \quad \text { iff } \quad x=y, \\
& d(x, z) \leq d(x, y)+d(y, z) \\
& d(x, y)=d(y, x)
\end{aligned}
$$

for all $x, y, z \in V$. Remember that each metric space $\langle V, d\rangle$ induces the interior operator $\mathbb{I}_{d}$ on $V$ : for all $X \subseteq V$,

$$
\mathbb{I}_{d}(X)=\{u \in X \mid \exists \varepsilon>0 \forall v(d(u, v)<\varepsilon \rightarrow v \in X)\} .
$$

$\left\langle V, \mathbb{I}_{d}\right\rangle$ is called the topological space induced by the metric space $\langle V, d\rangle$. The closure operator $\mathbb{C}_{d}$ in this space is dual to $\mathbb{I}_{d}$, that is, $\mathbb{C}_{d}(X)=V-\mathbb{I}_{d}(V-X)$; in other words,

$$
\mathbb{C}_{d}(X)=\{u \in V \mid \forall \varepsilon>0 \exists v \in X d(u, v)<\varepsilon\} .
$$

Thus, the intended models of the logic we are looking for are of the form

$$
\mathfrak{M}=\left\langle V, d, \mathbb{I}_{d}, P_{1}^{\mathfrak{M}}, P_{2}^{\mathfrak{M}}, \ldots\right\rangle,
$$

where $\left\langle V, d, P_{1}^{\mathfrak{M}}, P_{2}^{\mathfrak{M}}, \ldots\right\rangle$ is a metric model and $\mathbb{I}_{d}$ is the interior operator induced by $\langle V, d\rangle$. These structures will be called topometric models.

Language I. There are many approaches to devising languages that are capable of speaking about topometric models. The first natural choice would be the appropriate (fragment of) two-sorted first-order logic, with one sort for the elements of the metric space $\langle V, d\rangle$, another one for the distances in $\mathbb{R}^{+}$, and countably many unary predicates interpreted over the domain $V$, ternary predicates $d(x, y)<z$, where $x, y \in V, z \in \mathbb{R}^{+}$, and additional constants and operators like 0 and + with their standard interpretation over $\mathbb{R}$. However, as was shown in [26], even the two-variable fragment of monadic predicate logic with only one sort for the domain of the metric space and binary predicates $d(x, y)<a$, a a natural number between 0 and 80 , is undecidable. It was also proved in [26] that propositional operators 'somewhere in the open sphere of radius $a$ but not in its centre' give rise to an undecidable logic. On the other hand, we know from [35] that the (propositional) logic of metric spaces with operators 'somewhere in the open/closed sphere of radius $a^{\prime}$ is decidable and finitely axiomatisable. To speak about the topological component we take the standard language of $\mathbf{S 4}$ [29] extended with the universal and existential modalities over the space (which turned out to be very useful in spatial representation and reasoning $[7,1])$. 
Thus, the basic language $\mathcal{M T}$ we propose to use for speaking about topometric models is built from atomic terms $P_{i}, i<\omega$, with the help of the following rule:

$$
\tau \quad:=P_{i}|\neg \tau| \tau_{1} \sqcap \tau_{2}\left|\exists^{<a} \tau\right| \exists^{\leq a} \tau|\square \tau| \exists \tau,
$$

where $a$ is an arbitrary positive rational number, i.e., $a \in \mathbb{Q}^{+}$. We call $\tau$ an $\mathcal{M T}$-term or simply a term. We remind the reader of the definitions of standard 'topological fragments' of $\mathcal{M T}$. The language $\mathcal{M L}$ of $\mathbf{S} 4$ is defined by

$$
\tau \quad::=P_{i}|\neg \tau| \tau_{1} \sqcap \tau_{2} \mid \square \tau
$$

and the language $\mathcal{M} \mathcal{L}_{u}$ of $\mathbf{S} \boldsymbol{4}_{u}$ is

$$
\tau \quad::=P_{i} \quad|\neg \tau| \tau_{1} \sqcap \tau_{2}|\square \tau \quad| \quad \exists \tau .
$$

For a topometric model $\mathfrak{M}$ of the form (1), the extension $\tau^{\mathfrak{M}}$ of a term $\tau$ is computed inductively as follows:

$$
\begin{aligned}
\left(\tau_{1} \sqcap \tau_{2}\right)^{\mathfrak{M}} & =\tau_{1}^{\mathfrak{M}} \cap \tau_{2}^{\mathfrak{M}}, \\
\left(\neg \tau_{1}\right)^{\mathfrak{M}} & =V-\tau_{1}^{\mathfrak{M}}, \\
\left(\exists^{<a} \tau_{1}\right)^{\mathfrak{M}} & =\left\{u \in V \mid \exists v \in \tau_{1}^{\mathfrak{M}} d(u, v)<a\right\}, \\
\left(\exists^{\leq a} \tau_{1}\right)^{\mathfrak{M}} & =\left\{u \in V \mid \exists v \in \tau_{1}^{\mathfrak{M}} d(u, v) \leq a\right\}, \\
\left(\square \tau_{1}\right)^{\mathfrak{M}} & =\mathbb{I}_{d}\left(\tau_{1}^{\mathfrak{M}}\right), \\
\left(\exists \tau_{1}\right)^{\mathfrak{M}} & = \begin{cases}V & \text { if } \tau_{1}^{\mathfrak{M}} \neq \emptyset, \\
\emptyset & \text { otherwise. }\end{cases}
\end{aligned}
$$

Say that a term $\tau$ is satisfied in $\mathfrak{M}$ if $\tau^{\mathfrak{M}} \neq \emptyset ; \tau$ is true in $\mathfrak{M}$ if $\tau^{\mathfrak{M}}=V$. We denote by $\forall^{<a}, \forall \leq a, \diamond$, and $\forall$ the operators dual to $\exists^{<a}, \exists \leq a, \square$, and $\exists$, respectively. For instance,

$$
\left(\forall^{<a} \tau\right)^{\mathfrak{M}}=\left\{u \in V \mid \forall v \in V\left(d(u, v)<a \rightarrow v \in \tau^{\mathfrak{M}}\right)\right\}
$$

and $\diamond$ is interpreted by the closure operator $\mathbb{C}_{d}$.

The following examples illustrate the expressive capabilities of $\mathcal{M T}$.

EXAMPLE 1. (i) Set $\tau_{1} \sqcup \tau_{2}=\neg\left(\neg \tau_{1} \sqcap \neg \tau_{2}\right)$. Then the term $\forall\left(\neg \tau_{1} \sqcup \tau_{2}\right)$ is true in a topometric model $\mathfrak{M}$ if and only if $\tau_{1}^{\mathfrak{M}} \subseteq \tau_{2}^{\mathfrak{M}}$ holds in $\mathfrak{M}$. So we use the subsumption $\tau_{1} \sqsubseteq \tau_{2}$ as an abbreviation for $\forall\left(\neg \tau_{1} \sqcup \tau_{2}\right)$, and write $\tau_{1}=\tau_{2}$ for the conjunction of $\tau_{1} \sqsubseteq \tau_{2}$ and $\tau_{2} \sqsubseteq \tau_{1}$.

(ii) The terms $\tau=\square \tau, \tau=\diamond_{\tau}$ and $\tau=\diamond \square \tau$ mean then that (the extension of) $\tau$ is open, closed and regular closed, respectively. The exercises mentioned in the introduction can be formalised as follows:

$$
\square \exists^{<a} X=\exists^{<a} X \text { and } \diamond \exists^{<a} X \nsubseteq \exists^{\leq a} X .
$$

(iii) The topology induced by a finite metric space is trivial (every set is both open and closed). Thus, even the term $P \sqcap \diamond \neg P$ (which is clearly satisfiable, say, in every Euclidean space) is not satisfiable in any finite topometric model.

(iv) The Hausdorff distance between two closed sets is bounded by $a$ if every point of one set is within distance $a$ from some point in the other set. It is 
used, e.g., to measure the approximate matching between images [24]. We write $d_{H}\left(\tau_{1}, \tau_{2}\right) \leq a$ to abbreviate the conjunction

$$
\left(\diamond \tau_{1} \sqsubseteq \exists \leq a \diamond \tau_{2}\right) \sqcap\left(\diamond \tau_{2} \sqsubseteq \exists \leq a \diamond \tau_{1}\right)
$$

(which formalises the definition of Hausdorff distance); $d_{H}\left(\tau_{1}, \tau_{2}\right)=a$ is an abbreviation for

$$
\left(d_{H}\left(\tau_{1}, \tau_{2}\right) \leq a\right) \sqcap\left(\left(\diamond \tau_{1} \nsubseteq \exists^{<a} \diamond \tau_{2}\right) \sqcup\left(\diamond \tau_{2} \nsubseteq \exists^{<a} \diamond \tau_{1}\right)\right) .
$$

Note that, in contrast to the standard definition via infimum, $d_{H}\left(\tau_{1}, \tau_{2}\right)=a$ implies that there is a point in one set which is within distance $\geq a$ from any point in the other set.

(v) The min-distance between two sets is bounded by $a$ if there exists a point in one set which is within distance $\leq a$ from a point in the other set. This definition can be formalised as follows

$$
d_{\min }\left(\tau_{1}, \tau_{2}\right) \leq a \quad \text { iff } \quad \tau_{1} \sqcap \exists^{\leq a} \tau_{2} \neq \perp,
$$

where $\perp$ stands for the empty set, say, $P \sqcap \neg P$.

(vi) The $\mathcal{R} C C$-8 (aka Egenhofer-Franzosa) relations [16, 21] between nonempty regular closed sets can be expressed in the language $\mathcal{M} \mathcal{L}_{u}$ of $\mathbf{S} \mathbf{4}_{u}[7,31]$. So they are expressible in $\mathcal{M T}$ as well. The Hausdorff and min-distances introduced above suggest natural metric extensions of these relations.

(vii) $\mathcal{M T}$ is not compact in the sense that there is an infinite set $\Gamma$ of terms such that, for every finite $\Gamma^{\prime} \subseteq \Gamma$, there exists a model $\mathfrak{M}$ with $\bigcap_{\tau \in \Gamma^{\prime}} \tau^{\mathfrak{M}} \neq \emptyset$, but there exists no model $\mathfrak{M}$ for which $\bigcap_{\tau \in \Gamma} \tau^{\mathfrak{M}} \neq \emptyset$. An example is given by the set of terms $\{\neg \diamond P\} \cup\left\{\exists<\frac{1}{n} P \mid n \in \mathbb{N}^{+}\right\}$.

(viii) The term $\left(\diamond \tau_{1}=\tau_{2}\right) \sqcap \exists \tau_{2} \sqcap \neg \exists \square \tau_{1}$ says that $\tau_{1}$ is dense in a nonempty $\tau_{2}$, but has no interior.

Logics in language I. Given a class $\mathcal{M}$ of topometric models, let $L(\mathcal{M})$, the logic of $\mathcal{M}$, be the set of those $\mathcal{M T}$-terms that are true in all models from $\mathcal{M}$. By MT we denote the logic of the class of all topometric models. In this paper we investigate in detail the minimal logic $\mathbf{M T}$ as well as the logics of the real line and plane, $L(\mathbb{R})$ and $L\left(\mathbb{R}^{2}\right)$ (that is the logics of the classes of topometric models based on $\mathbb{R}$ and $\mathbb{R}^{2}$, respectively).

Note that the topological fragments of these logics are well known. The fragments of $\mathbf{M T}, L(\mathbb{R})$ and $L\left(\mathbb{R}^{2}\right)$ in the language $\mathcal{M L}$ coincide with the modal logic S4 [29], which is PSPACE-complete [27]. The fragment of MT in the language $\mathcal{M} \mathcal{L}_{u}$ is the modal logic $\mathbf{S} 4_{u}$, which is also PSPACE-complete [5]. The fragments of $L(\mathbb{R})$ and $L\left(\mathbb{R}^{2}\right)$ in $\mathcal{M} \mathcal{L}_{u}$ coincide with the logic of all connected topological spaces (induced by metric spaces); it can be obtained from $\mathbf{S} \boldsymbol{4}_{u}$ by adding the 'connectivity axiom' of [32]

$$
\exists \square P_{1} \sqcap \exists \square P_{2} \sqcap \forall\left(\square P_{1} \sqcup \square P_{2}\right) \quad \sqsubseteq \quad \exists\left(\square P_{1} \sqcap \square P_{2}\right) .
$$

Language II. In the language $\mathcal{M T}$ we can formalise metric relations, but we cannot compare two distances without specifying their absolute values. To enable reasoning about relations between distances, we extend $\mathcal{M T}$ with numerical variables. More precisely, let $\mathcal{M T}[\mathcal{V}]$ be the language that is defined similarly to $\mathcal{M T}$, with the only difference being that instead of the parameters from $\mathbb{Q}^{+}$we 
use variables from the list $\mathcal{V}=\left\{x_{0}, x_{1}, \ldots\right\}$. Given an assignment $\mathfrak{a}: \mathcal{V} \rightarrow \mathbb{Q}^{+}$ and an $\mathcal{M T}[\mathcal{V}]$-term $\tau$, we denote by $\tau^{\mathfrak{a}}$ the $\mathcal{M T}$-term that results from $\tau$ by replacing each $x_{i}$ with $\mathfrak{a}\left(x_{i}\right)$.

Let $\Gamma$ be a set of constraints for the variables in $\mathcal{V}$, say, a set of rational linear inequalities over $\mathcal{V}$ or a polynomial equation. An $\mathcal{M T}[\mathcal{V}]$-term $\tau$ is called satisfiable relative to $\Gamma$ if there exists an assignment $\mathfrak{a}$ such that it solves $\Gamma$ and $\tau^{\mathfrak{a}}$ is satisfiable in a topometric model. Here are two simple examples of constraint systems $\Gamma$ :

- $\Gamma$ consists of equalities $x_{i}=a_{i}$, where $a_{i} \in \mathbb{Q}^{+}$. In this case an $\mathcal{M T}[\mathcal{V}]$-term $\tau$ is satisfiable relative to $\Gamma$ iff $\tau^{\mathfrak{a}}$ is satisfiable for $\mathfrak{a}: x_{i} \mapsto a_{i}$.

- $\Gamma$ consists of strict inequalities $x_{0}<x_{1}, x_{1}<x_{2}$, etc. In this case no absolute value for variables is fixed.

EXAmPLE 2. (i) The terms $d_{H}\left(\tau_{1}, \tau_{2}\right)=x, d_{H}\left(\tau_{3}, \tau_{4}\right)=y$ under the constraint $x \geq 2 y$ say that the Hausdorff distance between $\tau_{1}$ and $\tau_{2}$ is at least two times larger than the Hausdorff distance between $\tau_{3}$ and $\tau_{4}$.

(ii) We can express incomplete knowledge about distances by using interval constraints $a_{1} \leq x \leq a_{2}$.

(iii) Comparative distance statements can be used within quantifiers, e.g., $\forall^{<x} \tau$ under the constraint $x=d_{H}\left(\tau_{1}, \tau_{2}\right)$.

In the remaining three sections of the paper we consider in turn the logic formalisms defined above.

§3. The logic MT. The purpose of this section is a comprehensive analysis of the logic MT. With every $\mathcal{M T}$-term $\tau$ we associate a finite set of axiom schemata from which $\tau$ is derivable iff $\tau \in \mathbf{M T}$. We also introduce a sound and complete relational semantics for $\mathcal{M T}$ with respect to which $\mathbf{M T}$ has the finite model property (remember that, according to Example 1 (iii), MT does not enjoy the finite model property relative to the intended topometric models). Using this relational semantics, we prove EXPTIME-completeness of MT (for binary coding of the parameters). Let us begin by introducing the notation we need.

Parameter set. Suppose that $M \subseteq \mathbb{Q}^{+}$is such that

(+) if $a, b \in M$ and $a+b \leq \gamma_{M}$, then $a+b \in M$,

(-) if $a, b \in M$ and $a-b>0$, then $a-b \in M$,

where $\gamma_{M}=\sup M$ if $M$ is bounded and $\gamma_{M}=\infty$ otherwise. Then $M$ is called a parameter set. We also let $\varepsilon_{M}=\inf M$.

Given an $\mathcal{M T}$-term $\tau$, we definite a finite parameter set $M[\tau] \subseteq \mathbb{Q}^{+}$containing all parameters from $\tau$. Suppose that the numerical parameters occurring in $\tau$ comprise the set

$$
N(\tau)=\left\{\frac{a_{1}}{b_{1}}, \ldots, \frac{a_{n}}{b_{n}}\right\} \subseteq \mathbb{Q}^{+},
$$

where $a_{i}, b_{i}$ are mutually prime for $1 \leq i \leq n$. Let $\gamma_{\tau}=\frac{a_{j}}{b_{j}}$ be the maximal number in $N(\tau)$ and let

$$
\varepsilon_{\tau}=1 /\left(b_{1} \times \cdots \times b_{n}\right),
$$




$$
\begin{aligned}
\xi_{\tau} & =a_{j} \times b_{1} \times \cdots \times b_{j-1} \times b_{j+1} \times \cdots \times b_{n}, \\
M[\tau] & =\left\{n \times \varepsilon_{\tau} \mid n \in \mathbb{N}^{+}, 1 \leq n \leq \xi_{\tau}\right\} .
\end{aligned}
$$

It is an easy exercise to check that $M[\tau]$ is closed under $(+)$ and $(-)$, and so is a finite parameter set. Note that the closure under $(+)$ and $(-)$ of a finite set of real numbers can be infinite.

Length of term. Let us agree on how to measure the length $\ell(\tau)$ of a term $\tau$. Given a positive rational number $c=a / b$, where $a$ and $b$ are relatively prime integers, let $\ell(c)$ denote the smallest natural number exceeding $1+\log _{2}(a+1)+$ $\log _{2}(b+1)$. Then the length $\ell(\tau)$ of a term $\tau$ is defined inductively in the usual way (say, as the number of subterms of $\tau$ ) with the only exception:

$$
\ell\left(\exists^{<c} \tau\right)=\ell\left(\exists^{\leq c} \tau\right)=\ell(\tau)+1+\ell(c) .
$$

In other words, the parameters in $\tau$ are assumed to be represented in binary. It is not hard to see that

$$
\log _{2}(|M[\tau]|) \leq \log _{2}\left(\prod_{i=1}^{n} a_{i} \times b_{i}\right) \leq \ell(\tau),
$$

where the $a_{i} / b_{i}$ are elements of $N(\tau)$. It follows that $|M[\tau]| \leq 2^{\ell(\tau)}$.

Axiom system. Given a parameter set $M$, denote by $A x_{\mathbf{M T}}[M]$ the following axiomatic system. First, it contains some set of axiom schemata and inference rules (say, modus ponens) of classical propositional logic. Second, it has the standard axiom schemata and rules of the minimal multi-modal logic $\mathbf{K}$ with the 'necessity operators' op of the form $\square, \forall, \forall<a, a \in M$, and $\forall \leq a, a \in M$, namely,

$$
o p(\tau \rightarrow \rho) \rightarrow(\text { op } \tau \rightarrow o p \rho) \text { and } \frac{\tau}{o p \tau},
$$

where $\tau \rightarrow \rho$ is an abbreviation for $\neg(\neg \tau \sqcap \rho)$. Third, to ensure that $\square$ is an S4-operator and $\forall$ is an S5-operator quantifying over the whole metric space, we include the well-known axiom schemata

$$
\square \tau \rightarrow \tau, \quad \square \tau \rightarrow \square \square \tau, \quad \forall \tau \rightarrow \tau, \quad \forall \tau \rightarrow \forall \forall \tau, \quad \tau \rightarrow \forall \exists \tau, \quad \forall \tau \rightarrow \forall \leq^{\leq a} \tau,
$$

for $a \in M$. Finally, the following axiom schemata govern the metric operators and their interaction with topology (here $a$ and $b$ range over $M$ ):

$$
\begin{aligned}
& \tau \rightarrow \forall \leq a_{\exists} \leq a_{\tau}, \\
& \tau \rightarrow \forall^{<a} \exists^{<a} \tau, \\
& \forall \leq{ }^{<} \tau \rightarrow \tau \text {, } \\
& \forall<a \rightarrow \tau, \\
& \exists^{<a} \tau \rightarrow \exists^{\leq a} \tau \text {, } \\
& \exists^{\leq a} \tau \rightarrow \exists^{<b} \tau, \quad \text { for } a<b, \\
& \exists^{\leq a} \exists^{\leq b} \tau \rightarrow \exists^{\leq a+b} \tau, \quad \text { if } a+b \in M \text {, } \\
& \exists^{\leq a} \exists^{<b} \tau \rightarrow \exists^{<a+b} \tau, \quad \text { if } a+b \in M \text {, } \\
& \exists^{<a} \exists^{<b} \tau \rightarrow \exists^{<a+b} \tau, \text { if } a+b \in M \text {, }
\end{aligned}
$$




$$
\begin{aligned}
& \diamond \tau \rightarrow \exists^{<a} \tau, \\
& \exists^{<a} \diamond \tau \rightarrow \exists^{<a} \tau .
\end{aligned}
$$

Axioms (3) and (4) ensure symmetry of metric, (5) and (6) guarantee the condition $d(x, x)=0$, and (9)-(11) the triangle inequality. Axioms (7) and (8) reflect the relation between open and closed spheres. (12) and (13) are the only axioms we need to grasp the interaction between metric and topology.

Relational semantics. An $\mathcal{M T}[M]$-frame, for a parameter set $M$, is a structure of the form

$$
\mathfrak{F}=\left\langle W, R,\left(D_{a}^{\leq}\right)_{a \in M},\left(D_{a}^{<}\right)_{a \in M}\right\rangle,
$$

where $W$ is a nonempty set and $R, D_{a}^{\leq}, D_{a}^{<}(a \in M)$ are binary relations on $W$ satisfying the following properties $(q o R)-\left(D^{<} R\right)$ for all $u, v, w \in W$ and $a, b \in M$ :

$(q \circ R) \quad R$ is reflexive and transitive (a quasi-order),

$(r s D) \quad D_{a}^{<}$and $D_{a}^{\leq}$are symmetric and reflexive,

$\left(D^{<} D^{\leq}\right) \quad D_{a}^{<} \subseteq D_{a}^{\leq}$,

$\left(D^{\leq}<\right) \quad$ if $u D_{a}^{\leq} v$ and $a<b$, then $u D_{b}^{<} v$,

$\left(\operatorname{tr} D^{\leq}\right) \quad$ if $u D_{a}^{\leq} v D_{b}^{\leq} w$ and $a+b \in M$, then $u D_{a+b}^{\leq} w$,

$\left(\operatorname{tr} D^{\leq} D^{<}\right) \quad$ if $u D_{a}^{\leq} v D_{b}^{<} w$ and $a+b \in M$, then $u D_{a+b}^{<} w$,

$\left(\operatorname{tr} D^{<} D^{\leq}\right) \quad$ if $u D_{a}^{<} v D_{b}^{\leq} w$ and $a+b \in M$, then $u D_{a+b}^{<} w$,

$\left(R D^{<}\right) \quad$ if $u R v$, then $u D_{a}^{<} v$ for all $a>0$,

$\left(D^{<} R\right) \quad$ if $u D_{a}^{<} v R w$, then $u D_{a}^{<} w$.

An $\mathcal{M T}[M]$-model is a structure

$$
\mathfrak{K}=\left\langle\mathfrak{F}, P_{1}^{\mathfrak{K}}, P_{2}^{\mathfrak{K}}, \ldots\right\rangle,
$$

where $\mathfrak{F}$ is an $\mathcal{M T}[M]$-frame of the form (14) and the $P_{i}^{\mathfrak{K}}$ are subsets of $W$. The value $\tau^{\mathfrak{K}}$ of an $\mathcal{M T}[M]$-term $\tau$ in $\mathfrak{K}$ is defined inductively: the values of the $P_{i}$ are given by the model, the Boolean cases are standard, and

$$
\begin{aligned}
\left(\exists^{<a} \tau\right)^{\mathfrak{K}} & =\left\{u \in W \mid \exists v \in \tau^{\mathfrak{K}} u D_{a}^{<} v\right\}, \\
\left(\exists^{\leq a} \tau\right)^{\mathfrak{K}} & =\left\{u \in W \mid \exists v \in \tau^{\mathfrak{K}} u D_{a}^{\leq} v\right\}, \\
(\square \tau)^{\mathfrak{K}} & =\left\{u \in W \mid \forall v \in W\left(u R v \rightarrow v \in \tau^{\mathfrak{K}}\right)\right\}, \\
(\exists \tau)^{\mathfrak{K}} & = \begin{cases}W & \text { if } \tau^{\mathfrak{K}} \neq \emptyset, \\
\emptyset & \text { otherwise. }\end{cases}
\end{aligned}
$$

We say that $\tau$ is satisfied in an $\mathcal{M T}[M]$-model $\mathfrak{K}$ if $\tau^{\mathfrak{K}} \neq \emptyset ; \tau$ is true in $\mathfrak{K}$ if $\tau^{\mathfrak{K}}=W$.

Throughout this paper we shall often use a number of simple properties of $\mathcal{M T}[M]$-frames that can be easily derived from conditions $(q o R)-\left(D^{<} R\right)$. We formulate them in the following lemma, where $s=x_{0} R D x_{n}$ is an arbitrary finite sequence of the form $x_{0} S_{0} x_{1} S_{1} \ldots S_{n-1} x_{n}$ such that each $S_{i}$ is one of $R, D_{a}^{<}$or $D_{a}^{\leq}$, for some $a \in M$, and the sum $a_{s}$ of the numerical parameters occurring in $s$ belongs to $M$ : 
LEMMA 3. (a) if $u D_{a}^{\leq} v$ and $a \leq b$, then $u D_{b}^{\leq} v$,

(b) if $u R v D_{a}^{\leq} w$ and $a<b$, then $u D_{b}^{<} w$,

(c) if $u D_{a}^{<} v D_{b}^{<} w$ and $a+b \in M$, then $u D_{a+b}^{<} w$,

(d) if $u D_{a}^{<} v$ and $u R w$, then $w D_{a}^{<} v$,

(e) if $u D_{a}^{<} v\left(\right.$ or $\left.v D_{a}^{<} u\right), s=v R D w$ and $a+a_{s} \in M$, then $u D_{a+a_{s}}^{<} w$,

(f) if $s=u R D v$ and $a=\varepsilon_{M}+a_{s} \in M$, then $u D_{a}^{<} v$,

(g) if $s=v R D w, s^{\prime}=v R D w^{\prime}$, and $a=\varepsilon_{M}+a_{s}+a_{s^{\prime}} \in M$, then $w D_{a}^{<} w^{\prime}$.

ProOF. We only show how to prove (b) and (g), and leave the remaining (equally easy) cases to the reader.

(b) Suppose that $u R v D_{a}^{\leq} w$ and $a<b$. By $\left(R D^{<}\right)$, we then have $u D_{\varepsilon_{M}}^{<} v D_{a}^{\leq} w$. Since $M$ is closed under $(-)$ and $(+), \varepsilon_{M} \leq b-a$ and $\varepsilon_{M}+a \in M$. It follows by $\left(\operatorname{tr} D^{<} D^{\leq}\right)$that $u D_{\varepsilon_{M}+a}^{<} w$. If $\varepsilon_{M}+a=b$ then we are done. And if $\varepsilon_{M}+a<b$, then we use $\left(D^{<} D^{\leq}\right)$and $\left(D^{\leq}<\right)$which also give $u D_{b}^{<} w$.

(g) Suppose that $s=v R D w, s^{\prime}=v R D w^{\prime}$, and $a=\varepsilon_{M}+a_{s}+a_{s^{\prime}} \in M$. By (f), $v D_{\varepsilon_{M}+a_{s}}^{<} w$, and so, by symmetry, $w D_{\varepsilon_{M}+a_{s}}^{<} v$, from which, by (e), we obtain $w D_{a}^{<} w^{\prime}$.

Remark 4. Intuitively, $u D_{a}^{\leq} v\left(u D_{a}^{<} v\right)$ in $\mathfrak{F}$ says that the distance between $u$ and $v$ is $\leq a$ (respectively, $<a) ; u R v$ means that $u$ belongs to the topological closure of $\{v\}$. In fact, the truth-condition for $\square$ means that $\square$ is interpreted by the interior operator $\mathbb{I}_{\mathfrak{G}}$ of the topological space $\mathfrak{T}_{\mathfrak{G}}=\left\langle W, \mathbb{I}_{\mathfrak{G}}\right\rangle$ induced by the quasi-order $\mathfrak{G}=\langle W, R\rangle$-i.e., by

$$
\mathbb{I}_{\mathfrak{G}}(X)=\{x \in X \mid \forall y \in W(x R y \rightarrow y \in X)\} .
$$

Such spaces are known as Aleksandrov spaces. Alternatively they can be defined as topological spaces where arbitrary (not only finite) intersections of open sets are open; for details see $[2,11]$.

The constraints on the relations in $\mathcal{M T}[M]$-frames reflect the connection between metric and topology: $(q o R)$ corresponds to the $\mathbf{S} 4$-axioms for $\square$, and $(r s D)-\left(D^{<} R\right)$ to axioms $(3)-(13)$. In fact, it is an easy exercise to check that the axioms of $A x_{\mathbf{M T}}[M]$ are true in all $\mathcal{M T}[M]$-models and that these models preserve the inference rules for every parameter set $M$. Note, however, that the topological interpretation of $R$ differs drastically from the topology induced by metric spaces: for example, in a metric space every singleton $\{x\}$ is closed, while every $\mathcal{M T}[M]$-frame with a non-trivial $R$ contains a non-closed singleton.

Given a term $\tau$, we write $A x_{\mathbf{M T}}[\tau]$ for $A x_{\mathbf{M T}}[M[\tau]]$ and $\mathcal{M T}[\tau]$ for $\mathcal{M T}[M[\tau]]$.

We are now in a position to formulate our main result about the basic logic MT of metric and topology:

THEOREM 5. (i) The following conditions are equivalent for every $\mathcal{M T}$-term $\tau$ :

1. $\tau \in \mathbf{M T}$.

2. $\tau$ is derivable in $A x_{\mathbf{M T}}\left[\mathbb{Q}^{+}\right]$.

3. $\tau$ is derivable in $A x_{\mathbf{M T}}[\tau]$.

4. $\tau$ is true in all $\mathcal{M T}[\tau]$-models.

5. $\tau$ is true in all finite $\mathcal{M T}[\tau]$-models.

(ii) The decision problem for $\mathbf{M T}$ is EXPTIME-complete. 
Before going into details of the proof, we separate the conceptually interesting part from the 'folklore' or trivial observations. Clearly, $(3) \Rightarrow(2) \Rightarrow(1)$. By Sahlqvist's completeness theorem (see, e.g., [9, Theorem 4.42]), we also have $(4) \Leftrightarrow(3)$. Indeed, $A x_{\mathbf{M T}}[\tau]$ can be regarded as a normal multi-modal logic with Sahlqvist axioms. So $A x_{\mathbf{M T}}[\tau]$ is determined by its Kripke frames which clearly coincide with $\mathcal{M T}[\tau]$-frames. In other words, a term $\tau$ is derivable in $A x_{\mathbf{M T}}[\tau]$ iff $\tau$ is true in all $\mathbf{M T}[\tau]$-models.

Thus, to complete the proof of (i), it remains to show the implications $(5) \Rightarrow$ (4) and $(1) \Rightarrow(5)$. The former one - which means that MT has the finite model property (fmp, for short) with respect to $\mathcal{M T}[M]$-models - as well as the EXPTIME-completeness of MT will be proved in Section 3.1. The core part of Theorem 5 is the latter implication to be proved in Section 3.2. It states that every term $\tau$ satisfiable in a finite $\mathcal{M T}[M]$-model (with $M[\tau] \subseteq M$ ) is satisfiable in a topometric model. Actually, we show that every finite $\mathcal{M T}[M]$-model is a pmorphic image (in the natural sense to be defined below) of some (almost always infinite) topometric model. Thus, $\mathcal{M T}[M]$-models can be regarded as a sort of partial descriptions of scenarios which can be realised in topometric models. In the algebraic setting, which is closer to Tarski's programme mentioned in the introduction, this can be reformulated as follows: every Boolean algebra with operators for $\square, \forall^{<a}, \forall \leq a(a \in M), \forall$, that is induced by a finite $\mathcal{M T}[M]$-frame, can be embedded into the Boolean algebra with operators induced by some topometric model. The reader familiar with algebraic semantics of modal logics and duality theory should not have problems with reformulating these results in the algebraic manner (consult, e.g., [20]).

Remark 6. It is to be noted that the axiomatic system $A x_{\mathrm{MT}}[\tau]$ is not complete if we do not include in it the axioms for all parameters from the closure of $N(\tau)$ under both $(+)$ and $(-)$. For example, $\diamond \exists^{\leq 2} P \sqsubseteq \exists^{<3} P$ is not derivable from the axioms formulated for $M=\{2,3\}$ only.

\subsection{The fmp and EXPTIME-completeness.}

TheOREM 7. (i) If an $\mathcal{M T}$-term $\tau$ is satisfiable in an $\mathcal{M T}[\tau]$-model, then it is satisfiable in a finite $\mathcal{M T}[\tau]$-model.

(ii) The satisfiability problem for $\mathcal{M T}$-terms in $\mathcal{M T}$-models is decidable in exponential time in the length of the term.

Proof. The proof of both (i) and (ii) is based on the standard elimination method used, e.g., for PDL [22]. Roughly, it works as follows. Given a term $\tau$, take a suitable closure $\operatorname{cl}(\tau)$ (the Fischer-Ladner closure in case of PDL) of the set of subterms of $\tau$, form a set $\Gamma_{\tau}$ of appropriate types over $c l(\tau)$, define appropriate relations for $\square, \forall^{<a}, \forall \leq a, a \in M[\tau]$, on $\Gamma_{\tau}$ and, finally, eliminate recursively all of those types from $\Gamma_{\tau}$ that contain terms of the form $\neg \square \rho, \neg \forall<a \rho$, or $\neg \forall \leq a \rho$ having no 'witnesses' in $\Gamma_{\tau}$ (i.e., properly located types with $\neg \rho$ ). Then we show that $\tau$ is satisfiable iff the elimination procedure terminates with a set containing a type with $\tau$. (This is done by proving that if $\tau$ is satisfiable then $\tau$ is satisfiable in a finite model constructed from the resulting set of types.) Finally, we show that this procedure is 'only' exponential in $\ell(\tau)$ by proving that $\Gamma_{\tau}$ is 'only' exponential in $\ell(\tau)$. 
Given the simple first-order form of the constraints on $\mathcal{M T}[\tau]$-models, it is not too difficult to implement this scheme for $\mathbf{M T}$ - provided that we use the unary coding of the numerical parameters. The main novelty of the proof is the additional ingredients required to devise an EXPTIME algorithm in $\ell(\tau)$, i.e., under binary coding.

Without loss of generality we may assume that $\tau$ is constructed using the Booleans, the metric operators $\forall<a, \forall \leq a$, the interior operator $\square$, and the universal quantifier $\forall$. Since $M[\tau]$ is exponential in $\ell(\tau)$, a naïve adoption of, say, the Fischer-Ladner closure would result in our case in $2^{2^{|\ell(\tau)|}}$ types. To avoid this, we add to the language new operators $\forall \leq a^{+}, a \in M[\tau]$, with the following semantics. Given an $\mathcal{M T}[\tau]$-model $\mathfrak{K}$ of the form (15), we have $x \in\left(\forall \leq a^{+} \rho\right)^{\mathfrak{K}}$ iff $y \in \rho^{\mathfrak{K}}$ whenever $s=x R D y$ and $a_{s} \leq a$, where $s=x R D y$ is an arbitrary finite sequence of the form $x S_{0} x_{1} S_{1} \ldots S_{n} y$ such that each $S_{i}$ is one of $R, D_{b}^{<}$or $D_{b}^{\leq}$, for some $b \in M[\tau]$, and the sum $a_{s}$ of the numerical parameters occurring in $s$ belongs to $M[\tau]$.

Denote by $\operatorname{sub}(\tau)$ the set of all subterms of $\tau$ and by $\operatorname{cl}(\tau)$ the closure under single negation of the set

$$
\begin{aligned}
& \operatorname{sub}(\tau) \cup\left\{\square \rho \mid \forall^{<b} \rho \in \operatorname{sub}(\tau) \text { or } \forall \leq b \rho \in \operatorname{sub}(\tau)\right\} \cup \\
& \left\{\forall^{<a} \rho, \forall \leq a \rho, \forall \leq a^{+} \rho \mid\left(\forall^{<b} \rho \in \operatorname{sub}(\tau) \text { or } \forall \leq b \rho \in \operatorname{sub}(\tau)\right) \text { and } a \in M[\tau]\right\} .
\end{aligned}
$$

Say that a subset $T$ of $\operatorname{cl}(\tau)$ is a $\tau$-type if it satisfies the following conditions, where $b \in M[\tau]$ :

$\left(t_{1}\right) \neg \rho \in T$ iff $\rho \notin T$, for all $\neg \rho \in \operatorname{cl}(\tau)$;

$\left(t_{2}\right) \rho_{1} \sqcap \rho_{2} \in T$ iff $\rho_{1}, \rho_{2} \in T$, for all $\rho_{1} \sqcap \rho_{2} \in \operatorname{cl}(\tau)$;

$\left(t_{3}\right)$ if $\square \rho \in T$ then $\rho \in T$;

$\left(t_{4}\right)$ if $\forall \leq a^{+} \rho \in T$ and $b \leq a$, then $\forall \leq b^{+} \rho, \forall \leq b \rho \in T$;

$\left(t_{5}\right)$ if $\forall \leq a \rho \in T$ and $b \leq a$, then $\forall \leq b \rho, \forall<b \rho \in T$;

$\left(t_{6}\right)$ if $\forall<a \rho \in T$ and $b \leq a$, then $\forall^{<b} \rho, \square \rho \in T$;

$\left(t_{7}\right)$ if $\forall<a \rho \in T$ and $a-\varepsilon_{\tau}>0$ then $\forall \leq\left(a-\varepsilon_{\tau}\right)^{+} \rho \in T$.

$\left(t_{8}\right)$ if $\forall \rho \in T$ then $\rho \in T$.

A typical example of a $\tau$-type is the set

$$
T(u)=\left\{\rho \in \operatorname{cl}(\tau) \mid u \in \rho^{\mathfrak{K}}\right\}
$$

generated by any point $u \in W$, where $\mathfrak{K}$ is an $\mathcal{M T}[\tau]$-model of the form (15).

LEMma 8. The number of distinct $\tau$-types does not exceed $2^{c \cdot \ell(\tau)^{2}}$, for some constant $c>0$.

Proof. Every $\tau$-type $T$ is uniquely determined by the set of subterms $\rho$ of $\tau$ it contains and, for every subterm $\rho$ of $\tau$, by the maximal $a \in M[\tau]$ such that $\forall \leq a^{+} \rho \in T$, the maximal $a \in M[\tau]$ such that $\forall \leq a \rho \in T$, the maximal $a \in M[\tau]$ such that $\forall<a \rho \in T$, and, finally, whether or not $\square \rho \in T$. This follows from the conditions $\left(t_{1}\right)$ and $\left(t_{4}\right)-\left(t_{6}\right)$ for $\tau$-types. Therefore, the number of distinct $\tau$-types does not exceed

$$
2^{|s u b(\tau)|} \times 2^{|s u b(\tau)|} \times|M[\tau]|^{3 \cdot|s u b(\tau)|} \leq 2^{2 \cdot \ell(\tau)} \times 2^{3 \cdot \ell(\tau)^{2}}
$$

as required 
Now we are going to show that, if $\tau$ is satisfiable at all, then a certain subset $\mathfrak{T}$ of the set of all $\tau$-types can serve as the domain of a model satisfying $\tau$. The points $u$ in this model will coincide with the $\tau$-types they generate. The relations $R$ and $D_{a}^{\leq}$and $D_{a}^{<}$in this model will be the restrictions to $\mathfrak{T}$ of the following relations $\rightarrow_{R}, \leftrightarrow_{\leq a}$ and $\leftrightarrow_{<a}$, for $a \in M[\tau]$, defined on the set of all $\tau$-types.

- Let $T_{1} \rightarrow_{R} T_{2}$ iff conditions (R1)-(R5) are satisfied:

(R1) if $\square \rho \in T_{1}$ then $\square \rho \in T_{2}$,

(R2) if $\forall \leq a^{+} \rho \in T_{1}$ then $\forall \leq a^{+} \rho \in T_{2}$,

(R3) if $\forall<a \rho \in T_{1}$ and $a>\varepsilon_{\tau}$ then $\forall \leq\left(a-\varepsilon_{\tau}\right)^{+} \rho \in T_{2}$,

(R4) if $\forall<\varepsilon_{\tau} \rho \in T_{1}$ then $\square \rho \in T_{2}$,

(R5) if $\forall<a \rho \in T_{2}$ then $\forall<a \rho \in T_{1}$.

- Let $T_{1} \leftrightarrow \leq a T_{2}$ iff the following four conditions hold for $i=1,2$, where $\overline{1}=2, \overline{2}=1$ and $a \in M[\tau]:$

( $\leq 1)$ if $\forall \leq a \rho \in T_{i}$ then $\rho \in T_{\bar{i}}$,

$(\leq 2)$ if $\forall \leq b \rho \in T_{i}$ and $b>a$ then $\forall \leq b-a \rho \in T_{\bar{i}}$,

$(\leq 3)$ if $\forall<b \rho \in T_{i}$ and $b>a$ then $\forall<b-a \rho \in T_{\bar{i}}$,

$(\leq 4)$ if $\forall \leq b^{+} \rho \in T_{i}$ and $b>a$ then $\forall \leq(b-a)^{+} \rho \in T_{\bar{i}}$.

- Let $T_{1} \leftrightarrow<a T_{2}$ iff, for $i=1,2$ :

$(<1)$ if $\forall<a \rho \in T_{i}$ then $\square \rho \in T_{\bar{i}}$,

$(<2)$ if $\forall<b \rho \in T_{i}$ and $b>a$ then $\forall \leq(b-a)^{+} \rho \in T_{\bar{i}}$.

Given a set $\mathcal{U}=\left\{\forall \tau_{1}, \ldots, \forall \tau_{r}\right\} \subseteq \operatorname{sub}(\tau)$, denote by $\mathcal{S}_{\mathcal{U}}$ the set of all $\tau$-types $T$ such that, for all subterms of $\tau$ of the form $\forall \rho$, we have

$$
\forall \rho \in T \quad \text { iff } \quad \forall \rho \in \mathcal{U} .
$$

That is to say, $T \in \mathcal{S}_{\mathcal{U}}$ if and only if the set of terms of the form $\forall \rho$ in $T$ coincides with $\mathcal{U}$. Clearly, the cardinality of the set of distinct $\mathcal{S}_{\mathcal{U}}$ is exponential in $\ell(\tau)$.

Now, to decide whether $\tau$ is satisfiable, we enumerate all $\mathcal{S}_{\mathcal{U}}$ and perform on each $\mathcal{S}_{\mathcal{U}}$ the following elimination procedure which checks whether it induces a model for $\tau$ or not. Form the sequence

$$
\mathcal{S}_{\mathcal{U}}=\mathcal{T}_{0} \supseteq \mathcal{T}_{1} \supseteq \cdots
$$

of sets of $\tau$-types as follows. Suppose $\mathcal{T}_{i}$ is defined. Delete all those types $T$ from $\mathcal{T}_{i}$ for which one of the following conditions holds:

- there is $\neg \forall \leq a \rho \in T$ such that there is no $T^{\prime} \in \mathcal{T}_{i}$ with $\neg \rho \in T^{\prime}$ and $T \leftrightarrow \leq a T^{\prime}$

- there is $\neg \forall<a \rho \in T$ such that there is no $T^{\prime} \in \mathcal{T}_{i}$ with $\neg \rho \in T^{\prime}$ and $T \leftrightarrow<a T^{\prime}$

- there is $\neg \forall \rho \in T$ such that there is no $T^{\prime} \in \mathcal{T}_{i}$ with $\neg \rho \in T^{\prime}$,

- there is $\neg \square \rho \in T$ such that there is no $T^{\prime} \in \mathcal{T}_{i}$ with $\neg \rho \in T^{\prime}$ and $T \rightarrow_{R} T^{\prime}$,

- there is some $\neg \forall \leq a^{+} \rho \in T$ for which there is no sequence $S=T \rightarrow R \leftrightarrow T^{\prime}$ of $\tau$-types such that $a_{S} \leq a$ and $\neg \rho \in T^{\prime}$ (where $S=T \rightarrow R \leftrightarrow T^{\prime}$ and $a_{S}$ are defined similarly to $s=x R D y$ and $a_{s}$ using the relations $\left.\rightarrow_{R}, \leftrightarrow \leq a, \leftrightarrow<b\right)$. Observe that, for every set $\mathcal{U}$ of the form $\left\{\forall \tau_{1}, \ldots, \forall \tau_{r}\right\} \subseteq \operatorname{sub}(\tau)$, this elimination procedure terminates after at most exponentially many in $\ell(\tau)$ steps. As there are at most exponentially many in $\ell(\tau)$ different $\mathcal{U}$, we can find out in 
exponential time whether for at least one $\mathcal{U}$ the elimination procedure applied to $\mathcal{S}_{\mathcal{U}}$ terminates with a set of $\tau$-types at least one of which contains $\tau$.

LEMMA 9. If $\tau$ is satisfiable then there exists a set $\mathcal{U}$ of the form

$$
\left\{\forall \tau_{1}, \ldots, \forall \tau_{r}\right\} \subseteq \operatorname{sub}(\tau)
$$

such that the elimination procedure applied to $\mathcal{S}_{\mathcal{U}}$ terminates with a set $\mathcal{T}$ of $\tau$-types at least one of which contains $\tau$.

Proof. Suppose $\tau$ is satisfied in a model

$$
\begin{aligned}
& \mathfrak{K}=\left\langle\mathfrak{F}, P_{1}^{\mathfrak{K}}, P_{2}^{\mathfrak{K}}, \ldots\right\rangle, \quad \text { where } \\
& \mathfrak{F}=\left\langle W, R,\left(D_{a}^{\leq}\right)_{a \in M[\tau]},\left(D_{a}^{<}\right)_{a \in M[\tau]}\right\rangle .
\end{aligned}
$$

Let

$$
\begin{aligned}
\mathcal{U} & =\left\{\forall \rho \in \operatorname{sub}(\tau) \mid \rho^{\mathfrak{K}}=W\right\}, \\
\mathcal{T}_{\mathfrak{K}} & =\{T(u) \mid u \in W\} .
\end{aligned}
$$

We show that the elimination procedure applied to $\mathcal{S}_{\mathcal{U}}$ terminates with some set $\mathcal{T} \supseteq \mathcal{T}_{\mathfrak{K}}$. Assume that the elimination procedure generates a sequence

$$
\mathcal{S}_{\mathcal{U}}=\mathcal{T}_{0} \supseteq \mathcal{T}_{1} \supseteq \cdots
$$

Then it is sufficient to prove that (i) $\mathcal{T}_{0} \supseteq \mathcal{T}_{\mathfrak{K}}$ and (ii) for all $i \geq 0$, if $\mathcal{T}_{i} \supseteq \mathcal{T}_{\mathfrak{K}}$, then $\mathcal{T}_{i+1} \supseteq \mathcal{T}_{\mathfrak{K}}$. Claim (i) follows from the observation that $\forall \rho \in T(u)$ if and only if $\rho^{\mathfrak{K}}=W$, for all $u \in W$ and $\forall \rho \in \operatorname{sub}(\tau)$. For (ii) we consider the five elimination rules for all $T=T(u)$ such that $u \in W$.

- Suppose that $\neg \forall \leq a \rho \in T(u)$. Then there exists $v \in W$ such that $u D_{a}^{\leq a} v$ and $v \notin \rho^{\mathfrak{K}}$, from which we obtain $T(u) \leftrightarrow \leq a T(v)$ and $\neg \rho \in T(v)$.

- Suppose $\neg \forall^{<a} \rho \in T(u)$. Then there is $v \in W$ such that $u D_{a}^{<a} v$ and $v \notin \rho^{\mathfrak{K}}$. Therefore, $T(u) \leftrightarrow<_{a} T(v)$ and $\neg \rho \in T(v)$.

- Suppose $\neg \forall \rho \in T(u)$. Then there is $v \in W$ such that $v \notin \rho^{\mathfrak{K}}$, and so $\neg \rho \in T(v)$.

- Suppose $\neg \square \rho \in T(u)$. Then there is $v \in W$ such that $u R v$ and $v \notin \rho^{\mathfrak{K}}$, from which $T(u) \rightarrow_{R} T(v)$ and $\neg \rho \in T(v)$.

- Suppose $\neg \forall \leq a^{+} \rho \in T(u)$. Then there exists a sequence $u S_{0} u_{1} S_{1} \ldots S_{n} u_{n}$ such that each $S_{i}$ is one of $R, D_{a}^{<}$or $D_{a}^{\leq}$, for some $a \in M[\tau]$ such that $a_{S} \leq a$, where $a_{S}$ is the sum of the numerical parameters of this sequence, and $u_{n} \notin \rho^{\mathfrak{K}}$. It follows that there is a sequence $S=T(u) \rightarrow R \leftrightarrow T\left(u_{n}\right)$ such that $a_{S} \leq a$ and $\neg \rho \in T\left(u_{n}\right)$.

This completes the proof of the lemma.

LEMma 10. Suppose that the elimination procedure starts on input $\mathcal{S}_{\mathcal{U}}$ and terminates with $\mathcal{T}$. If $\tau \in T$, for some $T \in \mathcal{T}$, then $\tau$ is satisfiable.

Proof. Define an $\mathcal{M} \mathcal{T}[\tau]$-model $\mathfrak{K}=\left\langle\mathfrak{F}, P_{1}^{\mathfrak{K}}, P_{2}^{\mathfrak{K}}, \ldots\right\rangle$ by taking

$$
\mathfrak{F}=\left\langle W, R,\left(D_{a}^{\leq}\right)_{a \in M[\tau]},\left(D_{a}^{<}\right)_{a \in M[\tau]}\right\rangle,
$$

where

- $W=\mathcal{T}$

- $P_{i}^{\mathfrak{K}}=\left\{T \in W \mid P_{i} \in T\right\}$, for $i<\omega$, 
- $T R T^{\prime}$ iff $T \rightarrow_{R} T^{\prime}$,

- $T D_{a}^{\leq} T^{\prime}$ iff $T \leftrightarrow<_{a} T^{\prime}$,

- $T D_{a}^{<} T^{\prime}$ iff $T \leftrightarrow<a T^{\prime}$.

We show that (i) $\mathfrak{F}$ is an $\mathcal{M T}[\tau]$-frame and that (ii) for every $\rho \in c l(\tau)$ and every $T \in W, T \in \rho^{\mathfrak{K}}$ iff $\rho \in T$.

Let us start with (i) and check conditions $(q o R)-\left(D^{<} R\right)$.

$(q \circ R)$ That $R$ (i.e., $\rightarrow_{R}$ ) is reflexive and transitive follows immediately from the definition, $\left(t_{6}\right)$ and $\left(t_{7}\right)$.

( $r s D$ ) That $D_{a}^{\leq}$(that is $\leftrightarrow \leq_{a}$ ) is reflexive and symmetric follows from the definition and $\left(t_{4}\right)-\left(t_{6}\right)$.

$\left(D^{<} D^{\leq}\right)$follows from the definition and $\left(t_{3}\right)-\left(t_{5}\right)$.

$(D \leq<)$ Let $T_{1} \leftrightarrow \leq a T_{2}$ and $a<b$. If $\forall<b \rho \in T_{1}$ then, by $(\leq 3), \forall<b-a \rho \in T_{2}$ and, by $\left(t_{6}\right), \square \rho \in T_{2}$. Thus we have $(<1)$. Now, if $\forall^{<c} \rho \in T_{1}$, for $c>b$, then by $(\leq 3), \forall<c-a \rho \in T_{2}$. As $c-b<c-a$, we can use $\left(t_{7}\right)$ (together with $\left(t_{4}\right),\left(t_{5}\right)$ ) and the definition of $M[\tau]$ to obtain $\forall \leq(c-b)^{+} \rho \in T_{2}$, which proves $(<2)$.

The properties $\left(\operatorname{tr} D^{\leq}\right),\left(\operatorname{tr} D^{\leq} D^{<}\right),\left(\operatorname{tr} D^{<} D^{\leq}\right),\left(R D^{<}\right)$are easy and left to the reader.

$\left(D^{<} R\right)$ Suppose $T_{0} \leftrightarrow<_{a} T_{1} \rightarrow_{R} T_{2}$. We need to show that $T_{0} \leftrightarrow<_{a} T_{2}$. If $\forall<a \rho \in T_{0}$ then $\square \rho \in T_{1}$, and so, by (R1), $\square \rho \in T_{2}$. If $\forall<b \rho \in T_{0}$ and $b>a$, then we have $\forall \leq(b-a)^{+} \rho \in T_{1}$. Therefore, by (R2), $\forall \leq(b-a)^{+} \rho \in T_{2}$. Now suppose that $\forall<a \rho \in T_{2}$. Then, by (R5), $\forall<a \rho \in T_{1}$, and so $\square \rho \in T_{0}$. Finally, if $\forall<b \rho \in T_{2}$ and $b>a$, then, by (R5), $\forall{ }^{<b} \rho \in T_{1}$, and hence $\forall \leq(b-a)^{+} \rho \in T_{0}$.

Now we show (ii) by induction on the construction of $\rho$. If $\rho$ is an atomic term, then (ii) follows from the definition. The steps for the Boolean connectives can be proved using conditions $\left(t_{1}\right)$ and $\left(t_{2}\right)$ for $\tau$-types. We now consider the 'modal' connectives.

Let $\rho=\square \rho_{0}$ and $T \notin\left(\square \rho_{0}\right)^{\mathfrak{K}}$. Then there exists $T^{\prime} \in W$ such that $T R T^{\prime}$ and $T^{\prime} \notin \rho_{0}^{\mathfrak{K}}$. By the induction hypothesis, $\rho_{0} \notin T^{\prime}$. But then, by (R2), $\square \rho_{0} \notin T$. Conversely, suppose $\square \rho_{0} \notin T$. T is not eliminable. Therefore, by the fourth elimination rule, there exists $T^{\prime} \in W$ such that $T \rightarrow_{R} T^{\prime}$ and $\rho_{0} \notin T^{\prime}$. By the induction hypothesis, $T^{\prime} \notin \rho_{0}^{\mathfrak{K}}$. Hence, $T \notin\left(\square \rho_{0}\right)^{\mathfrak{K}}$.

The case $\rho=\forall \leq a \rho_{0}$ can be proved using condition $(\leq 1)$ and the first elimination rule.

The case $\rho=\forall^{<a} \rho_{0}$ can be proved using condition $(<1)$, property $\left(t_{3}\right)$ of $\tau$-types, and the second elimination rule.

The case $\rho=\forall \rho_{0}$ can be proved using property $\left(t_{8}\right)$ of $\tau$-types, the fact that all $T \in W$ contain the same terms of the form $\forall \rho_{0}\left(\right.$ as $\left.\mathcal{S}_{\mathcal{U}} \supseteq W\right)$, and the third elimination rule.

This proves Theorem 7 .

Our next theorem establishes the corresponding lower bound even without using the interior and closure operators:

THEOREM 11. The following problems are EXPTIME-hard:

(i) decide whether an $\mathcal{M T}$-term built using only the Booleans and the operators $\forall \leq 1$ and $\forall$ belongs to $\mathbf{M T}$; 
(ii) decide whether an $\mathcal{M T}$-term built using only the Booleans and the operators $\forall \leq n, n \in \mathbb{N}^{+}$, belongs to $\mathbf{M T}$.

PRoOF. The proofs are by reduction of the global K-consequence relation that is known to be EXPTIME-hard [33]. We remind the reader that the language $\mathcal{L}_{\mathbf{K}}$ of modal logic $\mathbf{K}$ extends propositional logic (with propositional variables $\left.p_{1}, p_{2}, \ldots\right)$ by means of one unary operator $\diamond . \mathcal{L}_{\mathbf{K}}$ is interpreted in models of the form

$$
\mathfrak{N}=\left\langle V, S, p_{1}^{\mathfrak{N}}, p_{2}^{\mathfrak{N}}, \ldots\right\rangle,
$$

where $V$ is a nonempty set, $S \subseteq V \times V$ and $p_{i}^{\mathfrak{N}} \subseteq V$. The value $\varphi^{\mathfrak{N}} \subseteq V$ of an $\mathcal{L}_{\mathbf{K}}$-formula $\varphi$ in $\mathfrak{N}$ is defined inductively as follows:

- $(\varphi \wedge \psi)^{\mathfrak{N}}=\varphi^{\mathfrak{N}} \cap \psi^{\mathfrak{N}}$

- $(\neg \varphi)^{\mathfrak{N}}=V-\varphi^{\mathfrak{N}}$;

- $(\diamond \varphi)^{\mathfrak{N}}=\left\{v \in V \mid \exists w\left(v S w \wedge w \in \varphi^{\mathfrak{N}}\right)\right\}$.

Say that $\varphi_{1}$ follows globally from $\varphi_{2}$ and write $\varphi_{2} \vdash \varphi_{1}$ if, for every model $\mathfrak{N}$, $\varphi_{1}^{\mathfrak{N}}=V$ whenever $\varphi_{2}^{\mathfrak{N}}=V$. The problem of deciding whether $\varphi_{2} \vdash \varphi_{1}$ holds is EXPTIME-hard [33].

We define a translation $\sharp$ from $\mathcal{L}_{\mathbf{K}}$ into the set of $\mathcal{M T}$-terms built from the Booleans and the operator $\exists \leq 1$. For out translation, the important difference between models for $\mathcal{L}_{\mathbf{K}}$ and $\mathcal{M T}$-models for the operator $\exists \leq 1$ is that the $\diamond$ of $\mathcal{L}_{\mathbf{K}}$ is interpreted by an arbitrary relation $S$ while the relation $D_{1}^{\leq}$interpreting $\exists^{\leq 1}$ is symmetric and reflexive. Thus, the translation has to 'encode' an arbitrary relation $S$ by means of a symmetric and reflexive relation. We achieve this by interpreting the points of the model for $\mathcal{L}_{\mathbf{K}}$ by means of the extension $\tau_{0}^{\mathfrak{K}}$ of a term $\tau_{0}$ and using 'colours' $\tau_{1}^{\mathfrak{K}}$ and $\tau_{2}^{\mathfrak{K}}$ to encode the relation $S$. Going from $\tau_{0}^{\mathfrak{K}}$ to $\tau_{0}^{\mathfrak{K}}$ by traversing $\tau_{1}^{\mathfrak{K}}$ and then $\tau_{2}^{\mathfrak{K}}$ gives the required arbitrary relation $S$. More precisely, take atomic terms $Y_{0}, Y_{1}$ and define $\tau_{0}=Y_{0} \sqcap Y_{1}, \tau_{1}=Y_{0} \sqcap \neg Y_{1}$, $\tau_{2}=\neg Y_{0} \sqcap \neg Y_{1}$. Now we set inductively

$$
\begin{aligned}
p_{i}^{\sharp} & =P_{i} \sqcap \tau_{0}, \\
(\varphi \wedge \psi)^{\sharp} & =\varphi^{\sharp} \sqcap \psi^{\sharp}, \\
(\neg \varphi)^{\sharp} & =\neg \varphi^{\sharp} \sqcap \tau_{0}, \\
(\diamond \varphi)^{\sharp} & =\tau_{0} \sqcap \exists \leq 1\left(\tau_{1} \sqcap \exists \leq 1\left(\tau_{2} \sqcap \exists \leq 1\left(\tau_{0} \sqcap \varphi^{\sharp}\right)\right)\right) .
\end{aligned}
$$

(i) We show that for any $\varphi_{1}, \varphi_{2} \in \mathcal{L}_{\mathbf{K}}$,

$$
\varphi_{2} \vdash \varphi_{1} \quad \text { iff } \quad\left(\tau_{0} \wedge \forall\left(\tau_{0} \rightarrow \varphi_{2}^{\sharp}\right)\right) \rightarrow \varphi_{1}^{\sharp} \in \mathbf{M T} .
$$

The direction from left to right is easy and left to the reader. Conversely, suppose $\varphi_{2} \not \forall \varphi_{1}$ We may assume that $\varphi_{2}^{\mathfrak{N}}=V$ and $r \notin \varphi_{1}^{\mathfrak{N}}$, for a model $\mathfrak{N}=\left\langle V, S, p_{1}^{\mathfrak{N}}, \ldots\right\rangle$ such that $\langle V, S\rangle$ is an intransitive irreflexive tree with root $r$. Now, for any $x \in V-\{r\}$, take its predecessor $x_{p}$ and insert two points $(x, 1)$, $(x, 2)$ between $x$ and $x_{p}$. In other words, define an $\mathcal{M T}[\{1\}]$-frame

$$
\mathfrak{F}=\left\langle W, R, D_{1}^{\leq}, D_{1}^{<}\right\rangle
$$

by taking

$$
W=V \cup((V-\{r\}) \times\{1\}) \cup((V-\{r\}) \times\{2\}),
$$


$R$ and $D_{1}^{<}$to be the identity relation on $W$, and $D_{1}^{\leq}$the reflexive and symmetric closure of the relation containing, for any $x \in V-\{r\}$, the pairs $\left(x_{p},(x, 1)\right)$, $((x, 1),(x, 2))$, and $((x, 2), x)$. Define an $\mathcal{M T}[\{1\}]$-model $\mathfrak{K}$ based on $\mathfrak{F}$ by taking

$$
\begin{aligned}
P_{i}^{\mathfrak{K}} & =p_{i}^{\mathfrak{N}}, \\
Y_{0}^{\mathfrak{K}} & =V \cup((V-\{r\}) \times\{1\}), \\
Y_{1}^{\mathfrak{K}} & =V .
\end{aligned}
$$

It is not difficult to see that $r \in\left(\tau_{0} \wedge \forall\left(\tau_{0} \rightarrow \varphi_{2}^{\sharp}\right)\right)^{\mathfrak{K}}$ but $r \notin\left(\varphi_{1}^{\sharp}\right)^{\mathfrak{K}}$. Therefore, $\left(\tau_{0} \wedge \forall\left(\tau_{0} \rightarrow \varphi_{2}^{\sharp}\right)\right) \rightarrow \varphi_{1}^{\sharp} \notin \mathbf{M T}$.

(ii) Call an $\mathcal{L}_{\mathbf{K}}$-formula $\varphi$ valid if $\varphi^{\mathfrak{N}}=V$, for every model $\mathfrak{N}$. It is proved in [12] that, for any $\varphi_{1}, \varphi_{2} \in \mathcal{L}_{\mathbf{K}}$,

$$
\varphi_{2} \vdash \varphi_{1} \quad \text { iff } \quad\left(\varphi_{2} \wedge \square \varphi_{2} \wedge \cdots \wedge \square^{2^{\ell\left(\varphi_{1}\right)+\ell\left(\varphi_{2}\right)}} \varphi_{2}\right) \rightarrow \varphi_{1}
$$

is valid, where $\ell\left(\varphi_{i}\right)$ is the number of subformulas of $\varphi_{i}$. Now, it follows immediately from the reduction (i) that

$$
\varphi_{2} \vdash \varphi_{1} \quad \text { iff } \quad\left(\tau_{0} \sqcap \forall \leq 3 \cdot 2^{\left(\ell\left(\varphi_{1}\right)+\ell\left(\varphi_{2}\right)\right)}\left(\tau_{0} \rightarrow \varphi_{2}^{\sharp}\right)\right) \rightarrow \varphi_{1}^{\sharp} \in \mathbf{M T} .
$$

This proves (ii).

Note that, as follows from (i), the complexity of MT does not depend on whether we use binary or unary coding. This contrasts with real-time logics where, for example, LTL is PSPACE complete, but being extended with the operators 'after $n$ ticks of the clock,' $n$ coded in binary, it becomes an EXPSPACEcomplete logic (of the same expressive power, of course) [4]. Theorem 11 (ii) is of interest because it states that the universal modalities can be simulated by distance operators with sufficiently large parameters.

3.2. Representation theorem. Now we show how to prove the implication $(1) \Rightarrow(5)$. The proof actually extends a representation theorem of McKinsey and Tarski [29] (see also $[32,8,1]$ for more recent proofs) which they use to show the implication $(1) \Rightarrow(5)$ for the topological fragment $\mathcal{M L}$ of our language. To start with, we formulate their representation theorem for the metric space consisting of the real line $\mathbb{R}$ with the standard Euclidean metric. Let $\mathbb{I}_{\mathbb{R}}$ denote the standard interior operator on $\mathbb{R}$. For a quasi-order $\mathfrak{F}=\langle W, R\rangle$ and $X \subseteq W$, set

$$
\square X=\{u \in X \mid \forall v \in W(u R v \rightarrow v \in X)\} .
$$

A map $f$ from $\mathbb{R}$ onto $W$ is called a topological morphism from $\mathbb{R}$ to $\mathfrak{F}$ if, for all $X \subseteq W$,

$$
f^{-1}(\square X)=\mathbb{I}_{\mathbb{R}} f^{-1}(X) .
$$

A point $r \in W$ is called a root of $\mathfrak{F}$ if $W=\{w \mid r R w\}$.

THEOREM 12 (McKinsey and Tarski). If $\mathfrak{F}$ is a finite quasi-order with root $r$, then there exists a topological morphism $f$ from $\mathbb{R}$ to $\mathfrak{F}$ such that $f(0)=r$.

This result yields the following topological variant of the implication $(1) \Rightarrow(5)$ :

Corollary 13 (McKinsey and Tarski). If an $\mathcal{M L}$-term $\varphi$ is satisfiable in a finite rooted quasi-order, then it is satisfiable in $\left\langle\mathbb{R}, \mathbb{I}_{\mathbb{R}}\right\rangle$. 
We now introduce the concepts we need to extend this result (with $\mathbb{R}$ replaced by arbitrary metric spaces) to our language of metric and topology.

Let $\mathfrak{D}=\left\langle V, d, \mathbb{I}_{d}\right\rangle$ be a metric space with the induced interior operator $\mathbb{I}_{d}$. Let $\mathfrak{F}=\left\langle W, R,\left(D_{a}^{\leq}\right)_{a \in M},\left(D_{a}^{<}\right)_{a \in M}\right\rangle$ be an $\mathcal{M T}[M]$-frame, for some parameter set $M$. Define the operator $\square$ as in (16). We say that a surjective map $f: V \rightarrow W$ is an $M$-morphism from $\mathfrak{D}$ to $\mathfrak{F}$ if the following conditions are satisfied for all $x, y \in V$, all $a \in M$, and all $X \subseteq W$ :

(M1) $f^{-1}(\square X)=\mathbb{I}_{d} f^{-1}(X)$;

(M2) if $d(x, y) \leq a$ then $f(x) D_{a}^{\leq} f(y)$;

(M3) if $d(x, y)<a$ then $f(x) D_{a}^{<} f(y)$;

(M4) if $f(x) D_{a}^{\leq} f(y)$ then there exists a $z \in V$ such that $d(x, z) \leq a$ and $f(z)=f(y)$;

(M5) if $f(x) D_{a}^{<} f(y)$ then there exists a $z \in V$ such that $d(x, z)<a$ and $f(z)=f(y)$.

The following proposition is easy and left to the reader (it corresponds to the well-known p-morphism theorem from modal logic; see, e.g., [12, Theorem 3.15] or $[9$, Theorem 3.14$])$ :

Proposition 14. (i) Let $\mathfrak{M}=\left\langle\mathfrak{D}, P_{1}^{\mathfrak{M}}, P_{2}^{\mathfrak{M}}, \ldots\right\rangle$ be a topometric model and $\mathfrak{K}=\left\langle\mathfrak{F}, P_{1}^{\mathfrak{K}}, P_{2}^{\mathfrak{K}}, \ldots\right\rangle$ an $\mathcal{M} \mathcal{T}[M]$-model. If there is an $M$-morphism $f: \mathfrak{D} \rightarrow \mathfrak{F}$ such that $P_{i}^{\mathfrak{M}}=f^{-1}\left(P_{i}^{\mathfrak{K}}\right)$ for all $i<\omega$, then $\tau^{\mathfrak{M}}=f^{-1}\left(\tau^{\mathfrak{K}}\right)$ for every $\mathcal{M T}$-term $\tau$ with $M[\tau] \subseteq M$.

(ii) Suppose $\mathfrak{D}, \mathfrak{F}$ and $\tau$ are as above. If $\tau$ is satisfied in an $\mathcal{M T}[M]$-model based on $\mathfrak{F}$ and there is an $M$-morphism $f: \mathfrak{D} \rightarrow \mathfrak{F}$, then $\tau$ is satisfied in a topometric model based on $\mathfrak{D}$.

Thus, in view of (ii) above, to achieve our aim and show the implication $(1) \Rightarrow(5)$, it suffices to prove the following fundamental representation theorem:

THEOREM 15. Given a finite $\mathcal{M T}[M]$-frame

$$
\mathfrak{F}=\left\langle W, R,\left(D_{a}^{\leq}\right)_{a \in M},\left(D_{a}^{<}\right)_{a \in M}\right\rangle
$$

with a finite parameter set $M$, one can construct a (possibly infinite) metric space $\mathfrak{D}=\left\langle V, d, \mathbb{I}_{d}\right\rangle$ and an $M$-morphism $f: \mathfrak{D} \rightarrow \mathfrak{F}$.

Proof. The metric space $\mathfrak{D}$ is constructed by means of a sort of 'unravelling' of $\mathfrak{F}$ into a tree-like metric space. However, instead of taking the set $W^{+}$of all finite nonempty sequences over $W$ as the domain of the new space (which is the standard construction), we define the domain $V$ of $\mathfrak{D}$ as

$$
V=(\mathbb{R} \times W)^{+},
$$

i.e., as the set of nonempty sequences $\left(r_{1}, v_{1}\right) \ldots\left(r_{n}, v_{n}\right), n<\omega$, over $\mathbb{R} \times W$. Elements of $V$ are denoted by $\vec{x}, \vec{y}, \vec{z}$, etc., with end $(\vec{x})$ being the last element of $\vec{x}$. This generalisation of the standard unravelling construction by means of copies of the real line $\mathbb{R}$ allows us to represent the 'local topologies' within $\mathfrak{F}$. More precisely, for every $w \in W$, let $W_{w}=\{u \in W \mid w R u\}$ and $R_{w}=R \uparrow W_{w}$. The pair $\left\langle W_{w}, R_{w}\right\rangle$ is known as a subframe of the $\mathbf{S 4}$-frame $\langle W, R\rangle$ generated 
by $w$. Note that, since $\langle W, R\rangle$ is a quasi-order, it is possible that $W_{u}=W_{v}$ for $u \neq v$.

According to Theorem 12, for every subframe $\mathfrak{F}_{v}=\left\langle W_{v}, R_{v}\right\rangle$ of $\langle W, R\rangle$, there exists a surjective map $f_{v}: \mathbb{R} \rightarrow W_{v}$ such that, for all $X \subseteq W_{v}$,

$$
f_{v}^{-1}\left(\square_{v} X\right)=\mathbb{I}_{\mathbb{R}} f_{v}^{-1}(X) \quad \text { and } \quad f_{v}(0)=v .
$$

Here $\square_{v} X=\left\{w \in W_{v} \mid \forall u \in W_{v}\left(w R_{v} u \rightarrow u \in X\right)\right\}$. Notice, however, that $\square X=\square_{v} X$ for every $X \subseteq W_{v}$ because $\left\langle W_{v}, R_{v}\right\rangle$ is generated by $v$. Fix such a map $f_{v}$ for each $v \in W$. We are going to introduce a metric on $V$ in such a way that the map $f: V \rightarrow W$ defined by taking

$$
f(\vec{x}(r, v))=f_{v}(r)
$$

for all $\vec{x} \in V \cup\{\lambda\},(r, v) \in \mathbb{R} \times W$, is the required $M$-morphism. (Here $\lambda$ denotes the empty string.)

We begin by introducing a notion of $\mathfrak{F}$-distance $d_{\mathfrak{F}}\left(w, w^{\prime}\right)$ between $w, w^{\prime} \in W$. Let $M^{-}=\left\{a^{-} \mid a \in M\right\}$. Three cases are possible:

Case 1: if there is $a \in M$ such that $w D_{a}^{\leq} w^{\prime}$ but $w D_{a}^{<} w^{\prime}$ does not hold, then $d_{\mathfrak{F}}\left(w, w^{\prime}\right)=a$.

Case 2: if there is $a \in M$ such that $w D_{a}^{<} w^{\prime}$ but $w D_{b}^{\leq} w^{\prime}$ does not hold for any $b<a, b \in M$, then $d_{\mathfrak{F}}\left(w, w^{\prime}\right)=a^{-}$.

Case 3: if $w D_{a}^{\leq} w^{\prime}$ does not hold for any $a \in M$, then $d_{\mathfrak{F}}\left(w, w^{\prime}\right)=\infty$.

It is not hard to see that $d_{\mathfrak{F}}: W \times W \rightarrow M \cup M^{-} \cup\{\infty\}$ is a well-defined function.

In fact, the $\mathfrak{F}$-distance $d_{\mathfrak{F}}\left(w, w^{\prime}\right)$ is just a convenient way of speaking about the relations $D_{a}^{<}$and $D_{a}^{\leq}$. Indeed, $d_{\mathfrak{F}}\left(w, w^{\prime}\right)=a \in M$ means that the distance encoded by these two relations is exactly $a$, and $d_{\mathfrak{F}}\left(w, w^{\prime}\right)=a^{-}$means that this distance is between $a$ and $b$, where $b$ is the maximal number in $M$ such that $b<a$. In the latter case we cannot yet fix a number for the distance between $w$ and $w^{\prime}$.

With every pair $(r, v) \in \mathbb{R} \times W$ we associate a non-negative real number $o p(r, v)$ in the following way. Suppose that $f_{v}(r)=w$ and $W_{w} \varsubsetneqq W_{v}$ (equivalently, not $w R v)$. Then

$$
o p(r, v)=\inf \left\{\left|r-r^{\prime}\right| \mid r^{\prime} \notin f_{v}^{-1}\left(W_{w}\right)\right\} .
$$

As $\left\langle W_{w}, R_{w}\right\rangle$ is the subframe of $\left\langle W_{v}, R_{v}\right\rangle$ generated by $w$, we have

$$
W_{w}=\square_{w} W_{w}=\square_{v} W_{w}=\square W_{w} .
$$

Note that $o p(r, v)>0$ since $r \in f_{v}^{-1}\left(W_{w}\right)=f_{v}^{-1}\left(\square W_{w}\right)$, and so $f_{v}^{-1}\left(W_{w}\right) \varsubsetneqq \mathbb{R}$ is open. If $W_{w}=W_{v}$ then we set $o p(r, v)=\gamma_{M}$ (in particular, op $(0, v)=\gamma_{M}$ ). Intuitively, we need the number $o p(r, v)$ to ensure that points outside the interval $[r-o p(r, v), r+o p(r, v)]$ are 'sufficiently far away' from points in different copies of $\mathbb{R}$. Take some

$$
\varepsilon<\min \left\{\varepsilon_{M}, a_{1}+a_{2}-\gamma_{M} \mid a_{1}, a_{2} \in M, a_{1}+a_{2}>\gamma_{M}\right\}
$$


and assign to each element $\vec{x}$ of $V$ a real number $\operatorname{dist}(\vec{x})$ by taking inductively:

$$
\begin{aligned}
\operatorname{dist}((r, v)) & =\frac{1}{2} \min \{o p(r, v), \varepsilon\}, \\
\operatorname{dist}(\vec{x}(r, v)) & =\frac{1}{2} \min \{\operatorname{dist}(\vec{x}), \quad o p(r, v)\} .
\end{aligned}
$$

We are now in a position to define a metric function $d$ on $V$. The definition consists of five cases. The important ones are I and II, whereas the remaining cases ensure that we obtain a tree-like metric space by taking as distances between points the sum of the already defined distances over the shortest path connecting them. The maximal distance between any two points in $V$ will be $\gamma_{M}+\epsilon_{M}$. One can easily define a tree metric space without introducing this bound, but having it minimises the number of case distinctions required.

Case I. Suppose that $\vec{y}=\vec{x}\left(r_{1}, v\right)$ and $\vec{z}=\vec{x}\left(r_{2}, v\right)$ for some $r_{1}, r_{2} \in \mathbb{R}$ and some $\vec{x} \in V \cup\{\lambda\}$, where $\lambda$ is the empty string. Then

$$
d(\vec{y}, \vec{z})=\min \left\{\gamma_{M}+\varepsilon_{M},\left|r_{1}-r_{2}\right|\right\} .
$$

That is to say, on each copy of the real line $\mathbb{R}$, the metric function $d$ coincides with the Euclidean distance 'cut' at $\gamma_{M}+\epsilon_{M}$. Notice that neither the behaviour of the interior operator $\mathbb{I}_{\mathbb{R}}$ nor the behaviour of the operators $\exists<a$ and $\exists \leq a$, $a \in M$, are affected by 'cutting' the distance function at $\gamma_{M}+\epsilon_{M}$.

Case II. Suppose that $\vec{y}=\vec{x}\left(r_{1}, v\right)$. For each $w^{\prime} \in W$, we define $d\left(\vec{y}, \vec{y}\left(0, w^{\prime}\right)\right)$. This is the subtlest case in the definition of $d$ : given the point $r_{1}$ in a copy of $\mathbb{R}$ corresponding to $\left\langle W_{v}, R_{v}\right\rangle$, we define the distance $d$ between this point and the 0 s in the 'successor copies' of $\mathbb{R}$ corresponding to $\left\langle W_{w^{\prime}}, R_{w^{\prime}}\right\rangle$, for $w^{\prime} \in W$. Let $w=f_{v}\left(r_{1}\right)$. So $w$ is the point in $W_{v}$ to which $r_{1}$ corresponds. Thus, the distance $d\left(\vec{y}, \vec{y}\left(0, w^{\prime}\right)\right)$ should depend on the value of $d_{\mathfrak{F}}\left(w, w^{\prime}\right)$. The definition is clear for $d_{\mathfrak{F}}\left(w, w^{\prime}\right) \in M \cup\{\infty\}:$

If $d_{\mathfrak{F}}\left(w, w^{\prime}\right)=\infty$, then $d\left(\vec{y}, \vec{y}\left(0, w^{\prime}\right)\right)=\gamma_{M}+\varepsilon_{M}$.

If $d_{\mathfrak{F}}\left(w, w^{\prime}\right)=a$, then $d\left(\vec{y}, \vec{y}\left(0, w^{\prime}\right)\right)=a$.

Now suppose that $d_{\mathfrak{F}}\left(w, w^{\prime}\right)=a^{-}$. Then $d\left(\vec{y}, \vec{y}\left(0, w^{\prime}\right)\right)$ should be between $a$ and the maximal $b \in M$ such that $b<a$. In other words, it should be between $a-\epsilon_{M}$ and $a$. Second, we want to make sure that for $a=\epsilon_{M}$ the distance $d\left(\vec{y}, \vec{y}\left(0, w^{\prime}\right)\right)$ is at least $\frac{1}{2} \epsilon_{M}$ because points in other copies of $\mathbb{R}$ should not influence the topology on the present copy of $\mathbb{R}$. Finally, we have to ensure that for any $r^{\prime}$ which is on the same copy of $\mathbb{R}$ as $r_{1}$ and which corresponds to a $w^{\prime \prime} \in W_{v}-W_{w}$,

$$
\left|r_{1}-r^{\prime}\right|+d\left(\vec{y}, \vec{y}\left(0, w^{\prime}\right)\right)>a .
$$

We can ensure this by having $d\left(\vec{y}, \vec{y}\left(0, w^{\prime}\right)>a-o p\left(r_{1}, v\right)\right.$. Actually, a bit more is required. We have to ensure that this inequality also holds for sums of such distances over paths from the root of the constructed tree metric space to $\vec{y}$. Taking this into account, we end up with the definition $d\left(\vec{y}, \vec{y}\left(0, w^{\prime}\right)\right)=a-\frac{\operatorname{dist}(\vec{y})}{2}$.

We extend $d$ to the remaining pairs $\vec{x}, \vec{y} \in V \times V$ by taking the appropriate sums of the distances defined so far: 
Case III. Let $\vec{y}=\vec{x}\left(r_{1}, v_{1}\right)\left(r_{2}, v_{2}\right) \ldots\left(r_{n}, v_{n}\right)$ and $\operatorname{end}(\vec{x})=\left(r_{0}, v_{0}\right)$. Then

$$
\begin{aligned}
d(\vec{x}, \vec{y})= & \min \left\{\gamma_{M}+\varepsilon_{M},\right. \\
& \left.\sum_{i=1}^{n}\left|r_{i}\right|+\sum_{i=0}^{n-1} d\left(\vec{x}\left(r_{1}, v_{1}\right) \ldots\left(r_{i}, v_{i}\right), \vec{x}\left(r_{1}, v_{1}\right) \ldots\left(r_{i}, v_{i}\right)\left(0, v_{i+1}\right)\right)\right\} .
\end{aligned}
$$

Case IV. Suppose now that

$$
\begin{aligned}
& \vec{x}=\vec{z}\left(r_{0}, v_{0}\right)\left(r_{1}, v_{1}\right)\left(r_{2}, v_{2}\right) \ldots\left(r_{n}, v_{n}\right), \\
& \vec{y}=\vec{z}\left(r_{0}^{\prime}, v_{0}\right)\left(r_{1}^{\prime}, v_{1}^{\prime}\right)\left(r_{2}^{\prime}, v_{2}^{\prime}\right) \ldots\left(r_{m}^{\prime}, v_{m}^{\prime}\right)
\end{aligned}
$$

and either $r_{0} \neq r_{0}^{\prime}$ or $r_{0}=r_{0}^{\prime}$ and $v_{1} \neq v_{1}^{\prime}$. Then set

$$
d(\vec{x}, \vec{y})=\min \left\{\gamma_{M}+\varepsilon_{M},\left|r_{0}-r_{0}^{\prime}\right|+d\left(\vec{z}\left(r_{0}, v_{0}\right), \vec{x}\right)+d\left(\vec{z}\left(r_{0}^{\prime}, v_{0}\right), \vec{y}\right)\right\} .
$$

Case V. Finally, set $d(\vec{x}, \vec{y})=d(\vec{y}, \vec{x})$ whenever $d(\vec{y}, \vec{x})$ is defined, while $d(\vec{x}, \vec{y})$ is not defined yet. For the remaining undefined $d(\vec{x}, \vec{y})$ set $d(\vec{x}, \vec{y})=\gamma_{M}+\varepsilon_{M}$.

Straightforward yet tedious computations show that the defined function $d$ is indeed a metric on $V$ (this is left for the reader). Thus we have

LEMMA 16. $\langle V, d\rangle$ is a metric space.

Now define a map $f: V \rightarrow W$ by taking

$$
f(\vec{x}(r, v))=f_{v}(r)
$$

for all $\vec{x} \in V \cup\{\lambda\},(r, v) \in \mathbb{R} \times W$. To show that $f$ is an $M$-morphism, we require the following auxiliary lemma.

Lemma 17. Suppose that $\vec{y}=\vec{x}\left(r_{1}, v_{1}\right) \ldots\left(r_{n}, v_{n}\right)$ and end $(\vec{x})=\left(r_{0}, v_{0}\right)$. Suppose also that, for $0 \leq i \leq n-1$,

$$
d_{\mathfrak{F}}\left(f_{v_{i}}\left(r_{i}\right), v_{i+1}\right) \in\left\{a_{i}, a_{i}^{-}\right\} \subseteq M \cup M^{-}, \quad a=\sum_{0 \leq i<n} a_{i}, \quad b=a+\varepsilon_{M} .
$$

Let $\overrightarrow{x^{\prime}} \in V$ be the result of replacing the last element $\left(r_{0}, v_{0}\right)$ of $\vec{x}$ with $\left(r_{0}^{\prime}, v_{0}\right)$. Finally, suppose $d(\vec{x}, \vec{y}) \leq \gamma_{M}$. Then the following hold true:

(1) if $b \leq \gamma_{M}$, then $f(\vec{x}) D_{b}^{<} f(\vec{y})$ and $f\left(\overrightarrow{x^{\prime}}\right) D_{b}^{<} f(\vec{y})$,

(2) $d(\vec{x}, \vec{y})>a-\operatorname{dist}(\vec{x})$,

(3) $a \leq \gamma_{M}$,

(4) if $d_{\mathfrak{F}}\left(f(\vec{x}), v_{1}\right)=a_{0}^{-}$, then $f(\vec{x}) D_{a}^{<} f(\vec{y})$,

(5) $d(\vec{x}, \vec{y})>a-\frac{1}{2}$ op $\left(r_{0}, v_{0}\right)$ and $d(\vec{x}, \vec{y})>a-\frac{1}{2} \varepsilon$,

(6) $d\left(\overrightarrow{x^{\prime}}, \vec{y}\right)>a+\frac{1}{2} o p\left(r_{0}, v_{0}\right)$ if $\left|r_{0}^{\prime}-r_{0}\right| \geq o p\left(r_{0}, v_{0}\right)$,

(7) if $\left|r_{0}^{\prime}-r_{0}\right|<o p\left(r_{0}, v_{0}\right), w \in W$ and $c \in M$, then $f(\vec{x}) D_{c}^{<} w$ implies $f\left(\overrightarrow{x^{\prime}}\right) D_{c}^{<} w$.

Proof. (1) By the definition of $f$ and $d_{\mathfrak{F}}$, we have

$$
v_{0} R f(\vec{x}) D_{a_{0}}^{\leq} v_{1} R f_{v_{1}}\left(r_{1}\right) D_{a_{1}}^{\leq} v_{2} \ldots D_{a_{n-1}}^{\leq} v_{n} R f(\vec{y}) .
$$

So, by Lemma 3 (f), $f(\vec{x}) D_{b}^{<} f(\vec{y})$. And since $v_{0} R f\left(\overrightarrow{x^{\prime}}\right)$, by Lemma $3(\mathrm{~g})$, we obtain $f\left(\overrightarrow{x^{\prime}}\right) D_{b}^{<} f(\vec{y})$. 
(2) We have

$$
\sum_{0 \leq i<n} \frac{\operatorname{dist}\left(\vec{y}_{i}\right)}{2} \leq \sum_{0 \leq i<n} \frac{\operatorname{dist}(\vec{x})}{2^{i+1}}<\operatorname{dist}(\vec{x}),
$$

where $\vec{y}_{i}=\vec{x}\left(v_{1}, r_{1}\right) \ldots\left(v_{i}, r_{i}\right)$, for $0 \leq i<n$. Since $d(\vec{x}, \vec{y}) \leq \gamma_{M}$, we conclude, using the definition of $d$ in cases III and II, that

$$
d(\vec{x}, \vec{y}) \geq a-\sum_{0 \leq i<n} \frac{\operatorname{dist}\left(\vec{y}_{i}\right)}{2}
$$

and therefore $d(\vec{x}, \vec{y})>a-\operatorname{dist}(\vec{x})$.

(3) Suppose $a>\gamma_{M}$. Then $a-\varepsilon>\gamma_{M}$. It follows that $a-\operatorname{dist}(\vec{x})>\gamma_{M}$, contrary to $(2)$ and $d(\vec{x}, \vec{y}) \leq \gamma_{M}$.

(4) As $f(\vec{x}) D_{a_{0}}^{<} v_{1}$, by Lemma 3 (e) and (1) we then obtain $f(\vec{x}) D_{a}^{<} f(\vec{y})$.

(5) follows from $(2)$, since $\operatorname{dist}(\vec{x}) \leq \frac{1}{2} o p\left(r_{0}, v_{0}\right)$ and $\operatorname{dist}(\vec{x}) \leq \frac{1}{2} \varepsilon$.

(6) We have, by case IV and (5),

$$
\begin{aligned}
d\left(\overrightarrow{x^{\prime}}, \vec{y}\right) & =d(\vec{x}, \vec{y})+\left|r_{0}^{\prime}-r_{0}\right| \\
& \geq d(\vec{x}, \vec{y})+o p\left(r_{0}, v_{0}\right) \\
& >a-\frac{1}{2} o p\left(r_{0}, v_{0}\right)+o p\left(r_{0}, v_{0}\right) \\
& \geq a+\frac{1}{2} o p\left(r_{0}, v_{0}\right) .
\end{aligned}
$$

(7) Suppose $f_{v_{0}}\left(r_{0}\right)=u$. As $\left|r_{0}^{\prime}-r_{0}\right|<o p\left(r_{0}, v_{0}\right)$, we have $r_{0}^{\prime} \in f_{v_{0}}^{-1}\left(W_{u}\right)$. It follows that $f_{v_{0}}\left(r_{0}^{\prime}\right) \in W_{u}$, i.e., $f(\vec{x}) R f\left(\overrightarrow{x^{\prime}}\right)$. It remains to use Lemma $3(\mathrm{~d})$. $\dashv$

We are now in a position to prove that $f$ is an $M$-morphism from $\left\langle V, d, \mathbb{I}_{d}\right\rangle$ onto $\mathfrak{F}$. Obviously, $f$ is surjective.

(M1) Let $X \subseteq W$. Then

$$
\begin{aligned}
\vec{x}(r, v) \in \mathbb{I}_{d} f^{-1}(X) \quad \text { iff } & \\
& \text { iff } \quad \exists \varepsilon>0 \forall \vec{y} \in V(d(\vec{x}(r, v), \vec{y})<\varepsilon \rightarrow f(\vec{y}) \in X) \\
& \text { iff } \quad \exists \varepsilon \in\left(0, \varepsilon_{M} / 2\right) \forall \vec{y} \in V(d(\vec{x}(r, v), \vec{y})<\varepsilon \rightarrow f(\vec{y}) \in X) \\
& \text { iff } \quad \exists \varepsilon \in\left(0, \varepsilon_{M} / 2\right) \forall \vec{y} \in\{\vec{x}(s, v) \mid s \in \mathbb{R}\} \\
& \\
& \text { iff } \quad \vec{x}(r, v) \in \mathbb{I}_{d} f_{v}^{-1}(X) \quad(d(\vec{x}(r, v), \vec{y})<\varepsilon \rightarrow f(\vec{y}) \in X) \\
& \text { iff } \quad \vec{x}(r, v) \in f_{v}^{-1}(\square X) \\
& \text { iff } \quad \vec{x}(r, v) \in f^{-1}(\square X) .
\end{aligned}
$$

(Here $\left(0, \varepsilon_{M} / 2\right)=\left\{\varepsilon \in \mathbb{R} \mid 0<\varepsilon<\varepsilon_{M} / 2\right\}$.) Note that the fourth equivalence follows from the observation that the distance between any two points in different copies of $\mathbb{R}$ is at least $\frac{1}{2} \epsilon_{M}$. This was ensured by the definition of $d$ in case II.

(M4) Suppose that $f(\vec{x}) D_{a}^{\leq} f(\vec{y})$. Let $w=f(\vec{y})$. Then $d(\vec{x}, \vec{x}(0, w)) \leq a$ and $f(\vec{x}(0, w))=f_{w}(0)=w$. Thus, $z=\vec{x}(0, w)$ is as required.

(M5) Suppose that $f(\vec{x}) D_{a}^{<} f(\vec{y})$. Let $w=f(\vec{y})$. Then $d((\vec{x}), \vec{x}(0, w))<a$ and $f(\vec{x}(0, w))=f_{w}(0)=w$. Thus, $z=\vec{x}(0, w)$ is as required.

(M3) Suppose $d(\vec{x}, \vec{y})<a$. Consider all cases I-V. 
Case I. Suppose that $\vec{x}=\vec{z}\left(r_{1}, v\right)$ and $\vec{y}=\vec{z}\left(r_{2}, v\right)$ for some $\vec{z} \in V \cup\{\lambda\}$, $r_{1}, r_{2} \in \mathbb{R}$. Then $f(\vec{x})=f_{v}\left(r_{1}\right)$ and $f(\vec{y})=f_{v}\left(r_{2}\right)$. Therefore, $v R f(\vec{x})$ and $v R f(\vec{y})$. By Lemma $3(\mathrm{~g}), f(\vec{x}) D_{c}^{<} f(\vec{y})$ for all $c \in M$. In particular, $f(\vec{x}) D_{a}^{<} f(\vec{y})$.

Case II. Suppose $\vec{x}=\vec{z}\left(r_{1}, v\right)$ and $\vec{y}=\vec{z}\left(r_{1}, v\right)\left(0, v^{\prime}\right)$. Then $f(\vec{x}) D_{a}^{<} f(\vec{y})$ follows by definition.

Case III. By induction on $n$ we show that, for every $a \in M$, if $d(\vec{x}, \vec{y})<a$ and $\vec{y}=\vec{x}\left(r_{1}, v_{1}\right) \ldots\left(r_{n}, v_{n}\right)$, then $f(\vec{x}) D_{a}^{<} f(\vec{y})$. The case $n=0$ is clear.

Let $\vec{y}=\vec{x}\left(r_{1}, v_{1}\right) \ldots\left(r_{n}, v_{n}\right)\left(r_{n+1}, v_{n+1}\right)$, end $(\vec{x})=\left(r_{0}, v_{0}\right)$, and $d(\vec{x}, \vec{y})<a$, $a \in M$. We find $a_{i} \in M$ such that, for $0 \leq i \leq n, d_{\mathfrak{F}}\left(f_{v_{i}}\left(r_{i}\right), v_{i+1}\right) \in\left\{a_{i}, a_{i}^{-}\right\}$. Set $a^{\prime}=\sum_{0 \leq i \leq n} a_{i}$.

By Lemma 17 (5), we have $a>a^{\prime}-\varepsilon_{M}$ because

$$
a^{\prime}-\varepsilon_{M}<a^{\prime}-\frac{1}{2} \varepsilon<d(\vec{x}, \vec{y})<a .
$$

If $a \geq a^{\prime}+\varepsilon_{M}$ then, by Lemma $17(1), f(\vec{x}) D_{a}^{<} f(\vec{y})$, and we are done. So it suffices to consider the case $a=a^{\prime}$.

Let $f(\vec{x}) D_{a_{0}}^{<} v_{1}$. Then, by Lemma $17(4), f(\vec{x}) D_{a}^{<} f(\vec{y})$, and we are done. Suppose now that $f(\vec{x}) D_{a_{0}}^{<} v_{1}$ does not hold. Then $f(\vec{x}) D_{a_{0}}^{\leq} v_{1}, d\left(\vec{x}, \vec{x}\left(0, v_{1}\right)\right)=a_{0}$, and therefore $d\left(\vec{x}\left(0, v_{1}\right), \vec{y}\right)<a-a_{0}$. It follows that $d\left(\vec{x}\left(r_{1}, v_{1}\right), \vec{y}\right)<a-a_{0}$ and, by Lemma $17(6),\left|0-r_{1}\right|<o p\left(r_{1}, v_{1}\right)$. By the induction hypothesis, $f\left(\vec{x}\left(r_{1}, v_{1}\right) D_{a-a_{0}}^{<} f(\vec{y})\right.$. So, by Lemma $17(7), f\left(\vec{x}\left(0, v_{1}\right)\right) D_{a-a_{0}}^{<} f(\vec{y})$. But then $f(\vec{x}) D_{a_{0}}^{\leq} v_{1} D_{a-a_{0}}^{<} f(\vec{y})$, and by $\left(\operatorname{tr} D^{\leq} D^{<}\right)$, we obtain $f(\vec{x}) D_{a}^{<} f(\vec{y})$.

Case IV. Suppose now that

$$
\begin{aligned}
& \vec{x}=\vec{z}\left(r_{0}, v_{0}\right)\left(r_{1}, v_{1}\right)\left(r_{2}, v_{2}\right) \ldots\left(r_{n}, v_{n}\right), \\
& \vec{y}=\vec{z}\left(r_{0}^{\prime}, v_{0}\right)\left(r_{1}^{\prime}, v_{1}^{\prime}\right)\left(r_{2}^{\prime}, v_{2}^{\prime}\right) \ldots\left(r_{m}^{\prime}, v_{m}^{\prime}\right)
\end{aligned}
$$

and either $r_{0} \neq r_{0}^{\prime}$ or $r_{0}=r_{0}^{\prime}$ and $v_{1} \neq v_{1}^{\prime}$. Let

$$
\begin{gathered}
d(\vec{x}, \vec{y})=\left|r_{0}-r_{0}^{\prime}\right|+d\left(\vec{z}\left(r_{0}, v_{0}\right), \vec{x}\right)+d\left(\vec{z}\left(r_{0}^{\prime}, v_{0}\right), \vec{y}\right)<a \\
b_{1}=\sum_{0 \leq i<n} a_{i}, \quad b_{2}=\sum_{0 \leq i<m} a_{i}^{\prime},
\end{gathered}
$$

where

$$
d_{\mathfrak{F}}\left(f_{v_{i}}\left(r_{i}\right), v_{i+1}\right) \in\left\{a_{i}, a_{i}^{-}\right\}, \quad d_{\mathfrak{F}}\left(f_{v_{i}^{\prime}}\left(r_{i}^{\prime}\right), v_{i+1}^{\prime}\right) \in\left\{a_{i}^{\prime},\left(a_{i}^{\prime}\right)^{-}\right\}
$$

$\left(v_{0}^{\prime}=v_{0}\right)$. Set $b=b_{1}+b_{2}$. By Lemma $17(5)$,

$$
d\left(\vec{z}\left(r_{0}, v_{0}\right), \vec{x}\right)>b_{1}-\frac{1}{2} \varepsilon, \quad d\left(\vec{z}\left(r_{0}^{\prime}, v_{0}\right), \vec{y}\right)>b_{2}-\frac{1}{2} \varepsilon
$$

So, $d(\vec{x}, \vec{y})>b-\varepsilon>b-\varepsilon_{M}$. If $a \geq b+\varepsilon_{M}$, then $f(\vec{x}) S_{a} f(\vec{y})$, by Lemma $3(\mathrm{~g})$, since $f_{v_{0}}(0) R f\left(\vec{z}\left(r_{0}, v_{0}\right)\right)$ and $f_{v_{0}}(0) R f\left(\vec{z}\left(r_{0}^{\prime}, v_{0}\right)\right)$.

Thus, it suffices to consider the case $a=b$.

Let $d\left(\vec{z}\left(r_{0}, v_{0}\right), \vec{x}\right)<b_{1}$ and $\left|r_{0}-r_{0}^{\prime}\right|<o p\left(r_{0}, v_{0}\right)$. Then, by (III) above, $f\left(\vec{z}\left(r_{0}, v_{0}\right)\right) D_{b_{1}}^{<} f(\vec{x})$ and, by Lemma $17(7), f\left(\vec{z}\left(r_{0}^{\prime}, v_{0}\right)\right) D_{b_{1}}^{<} f(\vec{x})$. Therefore, by Lemma $3(\mathrm{e}), f(\vec{x}) D_{b}^{<} f(\vec{y})$.

The case $d\left(\vec{z}\left(r_{0}^{\prime}, v_{0}\right), \vec{y}\right)<b_{2},\left|r_{0}-r_{0}^{\prime}\right|<o p\left(r_{0}^{\prime}, v_{0}\right)$ is dual to the previous one. 
Let $\left|r_{0}-r_{0}^{\prime}\right| \geq o p\left(r_{0}, v_{0}\right)$ and $\left|r_{0}-r_{0}^{\prime}\right| \geq o p\left(r_{0}^{\prime}, v_{0}\right)$. We may assume that $o p\left(r_{0}, v_{0}\right) \geq o p\left(\overline{r_{0}^{\prime}}, v_{0}\right)$. Now, by Lemma $17(6)$,

$$
d\left(\vec{z}\left(r_{0}^{\prime}, v_{0}\right), \vec{x}\right)>b_{1}+\frac{1}{2} o p\left(r_{0}, v_{0}\right)
$$

and, by Lemma 17 (5),

$$
d\left(\vec{z}\left(r_{0}^{\prime}, v_{0}\right), \vec{y}\right)>b_{2}-\frac{1}{2} o p\left(r_{0}^{\prime}, v_{0}\right)
$$

Therefore, $d(\vec{x}, \vec{y})>b_{1}+b_{2}$, which is a contradiction.

The case $d\left(\vec{z}\left(r_{0}, v_{0}\right), \vec{x}\right) \geq b_{1}, d\left(\vec{z}\left(r_{0}^{\prime}, v_{0}\right), \vec{y}\right) \geq b_{2}$ leads to a contradiction as well.

Let $d\left(\vec{z}\left(r_{0}, v_{0}\right), \vec{x}\right) \geq b_{1}$ and $\left|r_{0}-r_{0}^{\prime}\right| \geq o p\left(r_{0}^{\prime}, v_{0}\right)$. Then $d\left(\vec{z}\left(r_{0}, v_{0}\right), \vec{y}\right) \geq b_{2}$, by Lemma $17(6)$, which is a contradiction.

The case $d\left(\vec{z}\left(r_{0}^{\prime}, v_{0}\right), \vec{x}\right) \geq b_{2},\left|r_{0}-r_{0}^{\prime}\right| \geq o p\left(r_{0}, v_{0}\right)$ is dual to the previous one.

Case $\mathrm{V}$ is trivial.

(M2) Suppose $d(\vec{x}, \vec{y}) \leq a$. Again we check cases I-V.

Case I. As we know, $\vec{x}=\vec{z}\left(r_{1}, v\right)$ and $\vec{y}=\vec{z}\left(r_{2}, v\right)$ imply $\vec{x} D_{a}^{<} \vec{y}$ for all $a \in M$. So, $\vec{x} D_{a}^{\leq} \vec{y}$ for all $a \in M$.

Case II follows from the definition.

Case III. We show by induction on $n$ that, for every $a \in M$, if $d(\vec{x}, \vec{y}) \leq a$ and $\vec{y}=\vec{x}\left(r_{1}, v_{1}\right) \ldots\left(r_{n}, v_{n}\right)$, then $f(\vec{x}) D_{a}^{\leq} f(\vec{y})$. The case $n=0$ is trivial. Suppose now that $\vec{y}=\vec{x}\left(r_{1}, v_{1}\right) \ldots\left(r_{n}, v_{n}\right)\left(r_{n+1}, v_{n+1}\right)$, end $(\vec{x})=\left(r_{0}, v_{0}\right)$ and $d(\vec{x}, \vec{y}) \leq$ $a \in M$. We can find $a_{i} \in M$ such that, for $0 \leq i \leq n, d_{\mathfrak{F}}\left(f_{v_{i}}\left(r_{i}\right), v_{i+1}\right) \in\left\{a_{i}, a_{i}^{-}\right\}$. Set $a^{\prime}=\sum_{0 \leq i \leq n} a_{i}$. As for (M3), it suffices to consider the case $a=a^{\prime}$.

Let $f(\vec{x}) D_{a_{0}}^{<} v_{1}$. Then, by Lemma $17(4), f(\vec{x}) D_{a}^{<} f(\vec{y})$. Therefore $f(\vec{x}) D_{a}^{\leq} f(\vec{y})$, and we are done.

Assume now that $f(\vec{x}) D_{a_{0}}^{<} v_{1}$ does not hold. Then we have $f(\vec{x}) D_{a_{0}}^{\leq} v_{1}$ and $d\left(\vec{x}, \vec{x}\left(0, v_{1}\right)\right)=a_{0}$. Therefore,

$$
d\left(\vec{x}\left(0, v_{1}\right), \vec{y}\right) \leq a-a_{0} .
$$

If $o p\left(r_{1}, v_{1}\right) \leq\left|r_{1}-0\right|$, then

$$
d\left(\vec{x}\left(0, v_{1}\right), \vec{y}\right)>a-a_{0}+\frac{1}{2} o p\left(r_{1}, v_{1}\right)
$$

(by Lemma $17(6)$ ) and

$$
d(\vec{x}, \vec{y}) \geq a+\frac{1}{2} o p\left(r_{1}, v_{1}\right)
$$

which is a contradiction.

Suppose now that $o p\left(r_{1}, v_{1}\right)>\left|r_{1}-0\right|$.

Let $r_{1}=0$. Then $d\left(\vec{x}\left(0, v_{1}\right), \vec{y}\right)=d\left(\vec{x}\left(r_{1}, v_{1}\right)\right) \leq a-a_{0}$, and by the induction hypothesis, $f\left(\vec{x}\left(0, v_{1}\right)\right)=f\left(\vec{x}\left(r_{1}, v_{1}\right)\right) D_{a-a_{0}}^{\leq} f(\vec{y})$. Then we have $f(\vec{x}) D_{a}^{\leq} f(\vec{y})$ by $\left(\operatorname{tr} D^{\leq}\right)$.

Let $r_{1} \neq 0$. As $d(\vec{x}, \vec{y}) \leq a$, we have $d\left(\vec{x}\left(r_{1}, v_{1}\right), \vec{y}\right)<a-a_{0}$. By (M3), $f\left(\vec{x}\left(r_{1}, v_{1}\right)\right) D_{a-a_{0}}^{<} f(\vec{y})$. By Lemma $17(7), f\left(\vec{x},\left(0, v_{1}\right)\right) D_{a-a_{0}}^{<} f(\vec{y})$. Therefore, by $\left(\operatorname{tr} D^{\leq} D^{<}\right), f(\vec{x}) D_{a}^{<} f(\vec{y})$, and so $f(\vec{x}) D_{a}^{\leq} f(\vec{y})$. 
Case IV. Suppose now that

$$
\begin{aligned}
& \vec{x}=\vec{z}\left(r_{0}, v_{0}\right)\left(r_{1}, v_{1}\right)\left(r_{2}, v_{2}\right) \ldots\left(r_{n}, v_{n}\right), \\
& \vec{y}=\vec{z}\left(r_{0}^{\prime}, v_{0}\right)\left(r_{1}^{\prime}, v_{1}^{\prime}\right)\left(r_{2}^{\prime}, v_{2}^{\prime}\right) \ldots\left(r_{m}^{\prime}, v_{m}^{\prime}\right)
\end{aligned}
$$

and either $r_{0} \neq r_{0}^{\prime}$ or $r_{0}=r_{0}^{\prime}$ and $v_{1} \neq v_{1}^{\prime}$. Let

$$
\begin{gathered}
d(\vec{x}, \vec{y})=\left|r_{0}-r_{0}^{\prime}\right|+d\left(\vec{z}\left(r_{0}, v_{0}\right), \vec{x}\right)+d\left(\vec{z}\left(r_{0}^{\prime}, v_{0}\right), \vec{y}\right) \leq a, \\
b_{1}=\sum_{0 \leq i<n} a_{i}, \quad b_{2}=\sum_{0 \leq i<m} a_{i}^{\prime},
\end{gathered}
$$

where

$$
d_{\mathfrak{F}}\left(f_{v_{i}}\left(r_{i}\right), v_{i+1}\right) \in\left\{a_{i}, a_{i}^{-}\right\}, \quad d_{\mathfrak{F}}\left(f_{v_{i}^{\prime}}\left(r_{i}^{\prime}\right), v_{i+1}^{\prime}\right) \in\left\{a_{i}^{\prime},\left(a_{i}^{\prime}\right)^{-}\right\}
$$

$\left(v_{0}^{\prime}=v_{0}\right)$. Set $b=b_{1}+b_{2}$. As in (M3), it suffices to consider the case $a=b$.

Let $d\left(\vec{z}\left(r_{0}, v_{0}\right), \vec{x}\right)<b_{1}$ and $\left|r_{0}-r_{0}^{\prime}\right|<o p\left(r_{0}, v_{0}\right)$. By (M3), $f(\vec{x}) D_{b}^{<} f(\vec{y})$, and so $f(\vec{x}) D_{a}^{\leq} f(\vec{y})$.

The case $d\left(\vec{z}\left(r_{0}^{\prime}, v_{0}\right), \vec{y}\right)<b_{2}$ and $\left|r_{0}-r_{0}^{\prime}\right|<o p\left(r_{0}^{\prime}, v_{0}\right)$ is dual to the previous one.

The case $\left|r_{0}-r_{0}^{\prime}\right| \geq o p\left(r_{0}, v_{0}\right)$ and $\left|r_{0}-r_{0}^{\prime}\right| \geq o p\left(r_{0}^{\prime}, v_{0}\right)$ leads to a contradiction as in (M3).

Let $d\left(\vec{z}\left(r_{0}, v_{0}\right), \vec{x}\right) \geq b_{1}$ and $d\left(\vec{z}\left(r_{0}^{\prime}, v_{0}\right), \vec{y}\right) \geq b_{2}$. Then $r_{0}=r_{0}^{\prime}, d\left(\vec{z}\left(r_{0}, v_{0}\right), \vec{x}\right)=$ $b_{1}$ and $d\left(\vec{z}\left(r_{0}^{\prime}, v_{0}\right), \vec{y}\right)=b_{2}$. So, by (III), we obtain $f\left(\vec{z}\left(r_{0}, v_{0}\right) D_{b_{1}}^{\leq} f(\vec{x})\right.$ and $f\left(\vec{z}\left(r_{0}, v_{0}\right)\right) D_{b_{2}}^{\leq} f(\vec{y}) . f(\vec{x}) D_{a}^{\leq} f(\vec{y})$ follows from the symmetry of $D_{b_{1}}^{\leq}$and $\left(\operatorname{tr} D^{\leq}\right)$.

Let $d\left(\vec{z}\left(r_{0}, v_{0}\right), \vec{x}\right) \geq b_{1}$ and $\left|r_{0}-r_{0}^{\prime}\right| \geq o p\left(r_{0}^{\prime}, v_{0}\right)$. Then, by Lemma $17(6)$,

$$
d\left(\vec{z}\left(r_{0}, v_{0}\right), \vec{y}\right) \geq b_{2}+\frac{1}{2} o p\left(r_{0}^{\prime}, v_{0}\right)
$$

which is a contradiction.

The case $d\left(\vec{z}\left(r_{0}^{\prime}, v_{0}\right), \vec{x}\right) \geq b_{2}$ and $\left|r_{0}-r_{0}^{\prime}\right| \geq o p\left(r_{0}, v_{0}\right)$ is dual to the previous one.

This completes the proof of Theorem 15.

Remark 18. It is worth noting that this result does not hold for infinite parameter sets. For example, take the $\mathcal{M T}\left[\mathbb{Q}^{+}\right]$-model

$$
\mathfrak{M}=\left\langle\{0,1\}, R,\left(D_{a}^{<}\right)_{a \in \mathbb{Q}^{+}},\left(D_{a}^{\leq}\right)_{a \in \mathbb{Q}^{+}}, P^{\mathfrak{M}}\right\rangle,
$$

where $R=\leq, D_{a}^{<}=D_{a}^{\leq}=\{0,1\} \times\{0,1\}, P^{\mathfrak{M}}=\{1\}$. Then the underlying frame of $\mathfrak{M}$ is not a $\mathbb{Q}^{+}$-morphic image of any topometric space, since otherwise the set $\{\neg \diamond P\} \cup\left\{\exists<\frac{1}{n} P \mid n \in \mathbb{N}^{+}\right\}$from Example 1 (vi) would be satisfiable.

\section{$\S 4$. The logics of the real line and plane.}

The logic of $\mathbb{R}$. Let us consider now the satisfiability problem for $\mathcal{M T}$-terms in topometric models based on the real line, i.e., the one-dimensional Euclidean space $\langle\mathbb{R}, d\rangle$ with $d\left(r, r^{\prime}\right)=\left|r-r^{\prime}\right|$. This problem can be shown to be decidable by a straightforward embedding into the quantitative monadic logic of order QMLO introduced in [23].

The language of QMLO is built from atoms $x_{1}<x_{2}, x_{1}=x_{2}, P_{i}(x)$, using the Booleans, first-order quantifiers and the following rule: if $\varphi(x)$ is a formula of QMLO with only one free (first-order) variable $x$, then 
- $(\exists x)_{>x_{0}}^{<x_{0}+1} \varphi::=\exists x\left(x_{0}<x<x_{0}+1 \wedge \varphi(x)\right)$,

- $(\exists x)_{<x_{0}}^{>x_{0}-1} \varphi::=\exists x\left(x_{0}-1<x<x_{0} \wedge \varphi(x)\right)$

are also formulas of QMLO. The following result is proved in [23]:

THEOREM 19 (Hirshfeld and Rabinovich). Validity of formulas of QMLO in $\mathbb{R}$ is decidable.

Hirshfeld and Rabinovich [23] also prove that

- $(\exists x)_{\geq x_{0}}^{\leq x_{0}+n} \varphi$ and $(\exists x)_{\substack{<\\<x_{0}}}^{\geq x_{0}-n} \varphi$,

- $(\exists x)_{\geq x_{0}}^{<x_{0}+n} \varphi$ and $(\exists x)_{\leq x_{0}}^{>x_{0}-n} \varphi$

are expressible by QMLO-formulas, for $n \in \mathbb{N}$.

To show that the satisfiability problem for $\mathcal{M T}$-terms over $\langle\mathbb{R}, d\rangle$ is decidable, we observe first that this problem is reducible to satisfiability of $\mathcal{M T}[\mathbb{N}]$-terms over $\langle\mathbb{R}, d\rangle$ (of course, this holds for satisfiability in many other classes of topometric models): given an $\mathcal{M T}[\mathbb{N}]$-term with parameters $\frac{a_{1}}{b_{1}}, \ldots, \frac{a_{n}}{b_{n}}$, replace any $\frac{a_{i}}{b_{i}}$ in $\tau$ by $a_{i} \times b_{1} \times \cdots \times b_{i-1} \times b_{i+1} \times \cdots \times b_{n}$. Then the resulting term is satisfiable if and only if $\tau$ is satisfiable.

Now define inductively a translation $\sharp$ from the set of $\mathcal{M T}[\mathbb{N}]$-terms into the language of QMLO by taking

$$
\begin{aligned}
P_{i}^{\sharp} & =P_{i}(x), \\
\left(\exists^{<n} \tau\right)^{\sharp} & =(\exists y)_{\geq x}^{<x+n} \tau^{\sharp}(y) \vee(\exists y)_{\leq x}^{>x-n} \tau^{\sharp}(y), \\
\left(\exists^{\leq n} \tau\right)^{\sharp} & =(\exists y)_{\geq x}^{\leq x+n} \tau^{\sharp}(y) \vee(\exists y)_{\leq x}^{\geq x-n} \tau^{\sharp}(y), \\
(\diamond \tau)^{\sharp} & =\forall y\left(y>x \rightarrow \exists z\left(x \leq z<y \wedge \tau^{\sharp}(z)\right)\right) \vee \\
(\exists \tau)^{\sharp} & =\exists y\left(x=x \wedge \tau^{\sharp}(y)\right) .
\end{aligned}
$$

It is readily seen that, for every $\mathcal{M T}[\mathbb{N}]$-term $\tau$, we have $a \in \tau^{\mathfrak{M}}$ for some $a \in \mathbb{R}$ and some topometric model $\mathfrak{M}$ over $\langle\mathbb{R}, d\rangle$ iff $\tau^{\sharp}[a]$ holds in some QMLO-model. Thus, we obtain from Theorem 19:

TheOrem 20. The logic $L(\mathbb{R})$ is decidable.

The computational complexity of $L(\mathbb{R})$ in unknown. It should be clear that the language of QMLO is more expressive than $\mathcal{M T}$. For example, the QMLOformula $\exists x(x>y \wedge P(x))$ cannot be expressed by means of an $\mathcal{M T}$-term (interpreted in $\langle\mathbb{R}, d\rangle$ ). More interestingly, by extending $\mathcal{M T}$ with the operators $\exists<a, a \in \mathbb{N}$, such that

$$
\left(\exists_{>0}^{<a} \tau\right)^{\mathfrak{M}}=\left\{u \in V \mid \exists v\left(0<d(u, v)<a \wedge v \in \tau^{\mathfrak{M}}\right)\right\},
$$

we obtain an undecidable logic over the class of all topometric models even without the topological operators [26]. However, when interpreted over $\langle\mathbb{R}, d\rangle$, the extended language gives rise to a decidable logic because it is clearly embeddable into QMLO. Thus, there are natural metric operators that are 'too expressive' on arbitrary metric spaces and yet 'harmless' on the real line.

On the other hand, it is known that the 'punctuality' operators $\exists=a$ ('at distance $=a^{\prime}$ ) yield undecidable logics on $\mathbb{R}$ (both with and without finite variability constraints on the interpretations) [3], but it is an open problem whether the 
extension of $\mathcal{M T}$ with the punctuality operators is decidable over the class of topometric models.

The logic of $\mathbb{R}^{2}$. The situation becomes quite different if we consider the satisfiability problem for $\mathcal{M T}$-terms in topometric model based on the $2 \mathrm{D}$ Euclidean space. We consider points of $\mathbb{R} \times \mathbb{R}$, i.e., pairs $\mathbf{x}=\left(x_{1}, x_{2}\right)$, where $x_{1}, x_{2} \in \mathbb{R}$, as standard Euclidean vectors of length

$$
\|\mathbf{x}\|=\sqrt{x_{1}^{2}+x_{2}^{2}} .
$$

A metric space $\langle W, d\rangle$ is a subspace of the Euclidean space $\mathbb{R} \times \mathbb{R}$ if there is an injective map $f: W \rightarrow \mathbb{R} \times \mathbb{R}$ such that, for any two $x, y \in W$,

$$
d(x, y)=\|f(x)-f(y)\| .
$$

THEOREM 21. The satisfiability problem for $\mathcal{M T}[\{1,2\}]$-terms (even containing no topological operators $\square$ and $\diamond)$ in topometric models over subspaces of $\mathbb{R} \times \mathbb{R}$ or over $\mathbb{R} \times \mathbb{R}$ itself is undecidable.

Proof. The proof is by reduction of the undecidable $\mathbb{Z} \times \mathbb{Z}$ tiling problem (see [10] and references therein), which is formulated as follows. Given a finite set $\mathcal{T}=\left\{T_{0}, \ldots, T_{l}\right\}$ of tile types (i.e., $1 \times 1$-squares $T_{i}$ with colours left $\left(T_{i}\right)$, $\operatorname{right}\left(T_{i}\right)$, up $\left(T_{i}\right)$, and $\operatorname{down}\left(T_{i}\right)$ on their edges), decide whether the grid $\mathbb{Z} \times \mathbb{Z}$ can be covered with tiles, each of a type from $\mathcal{T}$, in such a way that the colours of adjacent edges on adjacent tiles match, or, more precisely, whether there exists a function $f: \mathbb{Z} \times \mathbb{Z} \rightarrow \mathcal{T}$ such that, for all $n, m \in \mathbb{Z}$, we have

$$
\begin{aligned}
\operatorname{right}(f(n, m)) & =\operatorname{left}(f(n+1, m)), \\
u p(f(n, m)) & =\operatorname{down}(f(n, m+1)) .
\end{aligned}
$$

So suppose that a set $\mathcal{T}=\left\{T_{0}, \ldots, T_{l}\right\}$ of tile types is given. Our aim is to construct an $\mathcal{M T}[\{1,2\}]$-term $\tau_{\mathcal{T}}$ which is satisfiable in a subspace of $\mathbb{R}^{2}$ (or in $\mathbb{R}^{2}$ itself) iff $\mathcal{T}$ can tile $\mathbb{Z} \times \mathbb{Z}$.

Define $\tau_{\mathcal{T}}$ to be the conjunction of the following terms, where $X_{0}, \ldots, X_{3}$, $Y_{0}, \ldots, Y_{3}, Z_{0}, \ldots, Z_{l}$ are atomic terms, $0 \leq i, j \leq 3,0 \leq n, m, k \leq l$, and $+_{4}$ and $-{ }_{4}$ denote addition and subtraction modulo 4 :

$$
\begin{aligned}
& X_{0} \sqcap Y_{0} \neq \perp, \\
& X_{i} \sqcap Y_{j} \sqsubseteq \exists \leq 1\left(X_{i+{ }_{4} 1} \sqcap Y_{j}\right), \\
& X_{i} \sqcap Y_{j} \sqsubseteq \exists^{\leq 1}\left(X_{i-{ }_{4} 1} \sqcap Y_{j}\right), \\
& X_{i} \sqcap Y_{j} \sqsubseteq \exists^{\leq 1}\left(X_{i} \sqcap Y_{j+{ }_{4} 1}\right), \\
& X_{i} \sqcap Y_{j} \sqsubseteq \exists^{\leq 1}\left(X_{i} \sqcap Y_{j-{ }_{4} 1}\right), \\
& Y_{j} \sqsubseteq \forall^{<2} \neg Y_{j+{ }_{4} 2}, \\
& X_{i} \sqsubseteq \forall^{<2} \neg X_{i+{ }_{4} 2}, \\
& X_{i} \sqcap Y_{j} \sqsubseteq \prod_{\left(i^{\prime}, j^{\prime}\right) \neq(i, j)} \neg \exists^{<1}\left(X_{i^{\prime}} \sqcap Y_{j^{\prime}}\right), \\
& \bigsqcup_{i} \sqcap Y_{j}=\bigsqcup_{k \leq l} Z_{k},
\end{aligned}
$$




$$
\begin{gathered}
Z_{m} \sqsubseteq \forall^{<1} \neg Z_{n} \quad(\text { for } n \neq m), \\
X_{i} \sqcap Y_{j} \sqcap Z_{k} \sqsubseteq \forall^{\leq 1}\left(\neg X_{i+{ }_{4} 1} \sqcup \neg Y_{j} \sqcup \bigsqcup_{\operatorname{right}\left(T_{k}\right)=\operatorname{left}\left(T_{m}\right)} Z_{m}\right), \\
X_{i} \sqcap Y_{j} \sqcap Z_{k} \sqsubseteq \forall \leq 1\left(\neg X_{i} \sqcup \neg Y_{j+_{4} 1} \sqcup \bigsqcup_{u p\left(T_{k}\right)=\operatorname{down}\left(T_{m}\right)} Z_{m}\right) .
\end{gathered}
$$

The intended meaning of the conjuncts of $\tau_{\mathcal{T}}$ will become clear from the following consideration. Suppose that $\tau_{\mathcal{T}}$ is satisfied in a model

$$
\mathfrak{B}=\left\langle W, d, X_{0}^{\mathfrak{B}}, \ldots, X_{3}^{\mathfrak{B}}, Y_{0}^{\mathfrak{B}}, \ldots, Y_{3}^{\mathfrak{B}}, Z_{0}^{\mathfrak{B}}, \ldots, Z_{l}^{\mathfrak{B}}\right\rangle,
$$

where $W \subseteq \mathbb{R} \times \mathbb{R}$ and $d$ is the restriction of the Euclidean metric to $W$. The terms of the form $X_{i} \sqcap Y_{j}$, for $0 \leq i, j \leq 3$, will be used to simulate the grid $\mathbb{Z} \times \mathbb{Z}$ in $\langle W, d\rangle$ in the following way.

Let $A_{i}=X_{i}^{\mathfrak{B}}, B_{j}=Y_{j}^{\mathfrak{B}}$, for $0 \leq i, j \leq 3$. By (19), there is some $\mathbf{r} \in A_{0} \cap B_{0}$. Vector $\mathbf{r}$ will represent the point $(0,0)$ of $\mathbb{Z} \times \mathbb{Z}$. By $(20)$, there is a point $\mathbf{x}_{1} \in X_{1} \cap Y_{0}$ such that $d\left(\mathbf{r}, \mathbf{x}_{1}\right)=\left\|\mathbf{r}-\mathbf{x}_{1}\right\| \leq 1$. But in fact, by $(26), d\left(\mathbf{r}, \mathbf{x}_{1}\right)=1$. So we can regard $\mathbf{x}_{1}$ as representing $(1,0)$. Using again (20) and (26), we can find $\mathbf{x}_{2} \in X_{2} \cap Y_{0}$ such that $d\left(\mathbf{x}_{1}, \mathbf{x}_{2}\right)=1$. Now, observe that in view of $(25)$, $d\left(\mathbf{r}, \mathbf{x}_{2}\right) \geq 2$, while by the triangular inequality, $d\left(\mathbf{r}, \mathbf{x}_{2}\right) \leq 2$. Thus, $d\left(\mathbf{r}, \mathbf{x}_{2}\right)=2$ and we can regard $\mathbf{x}_{2}$ as representing $(2,0)$. Using the same kind of argument we can find $\mathbf{x}_{3} \in X_{3} \cap Y_{0}$ such that $d\left(\mathbf{x}_{2}, \mathbf{x}_{3}\right)=1$ and $d\left(\mathbf{x}_{1}, \mathbf{x}_{3}\right)=2$. Now, since the metric $d$ is Euclidean, we conclude that $d\left(\mathbf{r}, \mathbf{x}_{3}\right)=3$, and so $\mathbf{x}_{3}$ can represent $(3,0)$. Then we find $\mathbf{x}_{4} \in X_{0} \cap Y_{0}$ for which $d\left(\mathbf{r}, \mathbf{x}_{4}\right)=4$; that will be $(4,0)$. All four points $\mathbf{r}, \mathbf{x}_{1}, \mathbf{x}_{2}, \mathbf{x}_{3}$ lie on the same straight line. (Observe that if $\langle W, d\rangle$ is not assumed to be a subspace of $\mathbb{R}^{2}$ then we could have, say, $d\left(\mathbf{r}, \mathbf{x}_{3}\right)=1$ and $\mathbf{r}=\mathbf{x}_{4} \cdot$ )

More generally, it follows from (19), (20), (21), (25), and (26) that there exists a vector a such that $\|\mathbf{a}\|=1$ and, for every $i \leq 3$,

$$
\{\mathbf{r}+(4 n+i) \cdot \mathbf{a} \mid n \in \mathbb{Z}\} \subseteq A_{i} \cap B_{0}
$$

This gives us points of the form $\left(n^{\prime}, 0\right)$ from the grid $\mathbb{Z} \times \mathbb{Z}$.

Similarly, using (19), (22)-(24) and (26), we can find a vector $\mathbf{b}$ such that $\|\mathbf{b}\|=1$ and, for all $j \leq 3$,

$$
\{\mathbf{r}+(4 m+j) \cdot \mathbf{b} \mid m \in \mathbb{Z}\} \subseteq A_{0} \cap B_{j}
$$

which gives us points of the form $\left(0, m^{\prime}\right)$ from $\mathbb{Z} \times \mathbb{Z}$. Note that, according to (26), $\mathbf{a}$ and $\mathbf{b}$ are linearly independent.

We claim now that in general, for all $i, j \leq 3$,

$$
\{\mathbf{r}+(4 m+j) \cdot \mathbf{b}+(4 n+i) \cdot \mathbf{a} \mid m, n \in \mathbb{Z}\} \subseteq A_{i} \cap B_{j},
$$

which gives all points $\left(n^{\prime}, m^{\prime}\right)$ from $\mathbb{Z} \times \mathbb{Z}$. To prove this claim, we first show that, for $j \leq 3$,

$$
\{\mathbf{r}+(4 m+j) \cdot \mathbf{b}+\mathbf{a} \mid m \in \mathbb{Z}\} \subseteq A_{1} \cap B_{j} .
$$

We know that $\mathbf{r}+\mathbf{a} \in A_{1} \cap B_{0}$. Using the same argument as above, we can show that there is a vector $\mathbf{v}$ such that $\|\mathbf{v}\|=1$ and

$$
\{\mathbf{r}+(4 m+j) \cdot \mathbf{v}+\mathbf{a} \mid m \in \mathbb{Z}\} \subseteq A_{1} \cap B_{j} .
$$


So, we have to prove that $\mathbf{v}=\mathbf{b}$. Assume first that $\mathbf{v}$ and $\mathbf{b}$ are linearly independent. Since $\|\mathbf{v}\|=\|\mathbf{b}\|=1$, for every vector $\mathbf{x}$, there exist $n, m \in \mathbb{Z}$ such that

$$
\|n \cdot \mathbf{b}+m \cdot \mathbf{v}-\mathbf{x}\|<1
$$

It follows then from (31) and (34), that there are $\mathbf{c} \in A_{0} \cap B_{j}$ and $\mathbf{d} \in A_{1} \cap B_{j^{\prime}}$, for some $j, j^{\prime} \leq 3$, such that $\|\mathbf{c}-\mathbf{d}\|<1$, contrary to (26). Therefore, $\mathbf{b}$ and $\mathbf{v}$ are linearly dependent. So it remains to show $\mathbf{b} \neq-\mathbf{v}$. Suppose otherwise. But then $\mathbf{r}+\mathbf{b} \in A_{0} \cap B_{1}$ and $\mathbf{r}+\mathbf{a}+\mathbf{b} \in A_{1} \cap B_{3}$, contrary to (24). (Here we again used the fact that the metric is Euclidean. Without it we could not ensure, for example, the existence of a point $\mathbf{c} \in A_{1} \cap B_{1}$ such that $d(\mathbf{r}+\mathbf{a}, \mathbf{c})=d(\mathbf{r}+\mathbf{b}, \mathbf{c})=1$.)

Thus we have (33) and then obtain (32) by a straightforward induction.

This provides us with an encoding of the grid $\mathbb{Z} \times \mathbb{Z}$. We use the atomic terms $Z_{i}$ to encode the tile types $T_{i}$ : (27) says that every point on the grid is covered with a tile, (28) ensures that this tile is unique, while (29) and (30) guarantee that the colours of adjacent edges on adjacent tiles match.

Consider now a map $f: \mathbb{Z} \times \mathbb{Z} \rightarrow \mathcal{T}$ defined by taking, for all $n, m \in \mathbb{Z}$ and $i, j \leq 3$,

$$
f(4 n+i, 4 m+j)=T_{k} \quad \text { iff } \quad \mathbf{r}+(4 n+i) \mathbf{a}+(4 m+j) \mathbf{b} \in Z_{k}^{\mathfrak{B}} .
$$

It is not hard to check that $f$ is a tiling we need.

Conversely, suppose that $f: \mathbb{Z} \times \mathbb{Z} \rightarrow \mathcal{T}$ tiles $\mathbb{Z} \times \mathbb{Z}$. Consider the model $\mathfrak{B}$ based on the Euclidean metric space $\mathbb{R} \times \mathbb{R}$ in which, for $0 \leq i \leq 3$ and $0 \leq k \leq l$,

$$
\begin{aligned}
X_{i}^{\mathfrak{B}} & =\{(i+4 n, m) \mid(n, m) \in \mathbb{Z} \times \mathbb{Z}\}, \\
Y_{i}^{\mathfrak{B}} & =\{(m, i+4 n) \mid(n, m) \in \mathbb{Z} \times \mathbb{Z}\}, \\
Z_{k}^{\mathfrak{B}} & =\left\{(n, m) \mid f(n, m)=T_{k}\right\} .
\end{aligned}
$$

It is readily checked that $\left(\tau_{\mathcal{T}}\right)^{\mathfrak{B}} \neq \emptyset$.

§5. Reasoning with numerical variables. The main aim of this section is to show that reasoning with the language $\mathcal{M T}[V]$ (containing numerical variables that satisfy some rational linear inequalities) is decidable:

THEOREM 22. It is decidable whether an $\mathcal{M T}[V]$-term $\tau$ is satisfiable relative to a finite set of rational linear inequalities.

To formulate the decision procedure, we require the following notation. Suppose that an $\mathcal{M T}[V]$-term $\tau$ and a set $\Gamma$ of rational linear inequalities over $\mathcal{X}=\left\{x_{1}, \ldots, x_{n}\right\}$ are given. Without loss of generality we may assume that $\Gamma$ contains (among others) the constraints

$$
\left\{0<x_{1}, x_{1}<x_{2}, x_{2}<x_{3}, \ldots, x_{n-1}<x_{n}\right\} .
$$

(Obviously, the general decidability problem can be reduced to $n$ ! decidability problems of this type.) Denote by $\mathcal{S}_{\Gamma}$ the set of all assignments $\mathfrak{a}: \mathcal{X} \rightarrow \mathbb{Q}^{+}$ solving $\Gamma$. Our problem is to decide whether there is $\mathfrak{a} \in \mathcal{S}_{\Gamma}$ such that $\tau^{\mathfrak{a}}$ is satisfiable. Since $\mathcal{S}_{\Gamma}$ is usually infinite, it is not clear a priori that this can be done effectively. The main step towards an algorithm solving this problem is to show that if there exists an $\mathfrak{a} \in \mathcal{S}_{\Gamma}$ such that $\tau^{\mathfrak{a}}$ is satisfiable, then there exists an 
assignment which comes from a finite and effectively computable set of solutions to $\Gamma$.

Intuitively, the finite set of solutions $\mathfrak{a}$ we need consists of those solutions that minimise the number of constraints on $\mathcal{M T}\left[\tau^{\mathfrak{a}}\right]$-models. More precisely, given an assignment $\mathfrak{a}$, denote by $L(\mathfrak{a})$ the set of all inequalities of the form

$$
\sum_{x_{i} \in Z} k_{x_{i}} x_{i} \leq x_{j} \quad \text { and } \quad \sum_{x_{i} \in Z} k_{x_{i}} x_{i}<x_{j}
$$

where the $k_{x_{i}}$ are positive natural numbers, $Z \subseteq \mathcal{X}, x_{j} \in \mathcal{X}$, and

$$
\sum_{x_{i} \in Z} k_{x_{i}} \mathfrak{a}\left(x_{i}\right) \leq \mathfrak{a}\left(x_{j}\right) \quad \text { or } \quad \sum_{x_{i} \in Z} k_{x_{i}} \mathfrak{a}\left(x_{i}\right)<\mathfrak{a}\left(x_{j}\right)
$$

respectively.

Each $L(\mathfrak{a})$ is a finite set of rational linear inequalities. However, there may exist infinitely many $L(\mathfrak{a}), \mathfrak{a} \in S_{\Gamma}$. But it turns out that the set of $\subseteq$-minimal members of $\left\{L(\mathfrak{a}) \mid \mathfrak{a} \in S_{\Gamma}\right\}$ is finite and provides enough information. Let

$$
\mathcal{M}_{\Gamma}=\left\{L(\mathfrak{a}) \mid \mathfrak{a} \in \mathcal{S}_{\Gamma} \& \neg \exists \mathfrak{b} \in \mathcal{S}_{\Gamma} L(\mathfrak{b}) \varsubsetneqq L(\mathfrak{a})\right\} .
$$

Lemma 23. $\mathcal{M}_{\Gamma}$ is finite and can be computed effectively from $\Gamma$.

Proof. Finiteness follows from the observation that

$$
\left\langle\left\{L(\mathfrak{a}) \mid \mathfrak{a} \in \mathcal{S}_{\Gamma}\right\}, \subseteq\right\rangle
$$

is a well partial order (it is easily embeddable into an appropriate $\left(\mathbb{N}^{m}, \leq\right.$ ), where $\left(n_{1}, \ldots, n_{m}\right) \leq\left(n_{1}^{\prime}, \ldots, n_{m}^{\prime}\right)$ iff $n_{i} \leq n_{i}^{\prime}$ for all $i \leq m$.) Recall that it can be checked effectively whether, for a given $\mathfrak{a}, \Gamma \cup L(\mathfrak{a})$ is solvable. If the answer is 'yes' then a solution can be computed. So, we can check effectively (i) whether $L(\mathfrak{a})$ is a member of $\mathcal{M}_{\Gamma}$, for a given $\mathfrak{a}$, and (ii) whether $X=\mathcal{M}_{\Gamma}$ holds, for a given $X \subseteq \mathcal{M}_{\Gamma}$.

Now the algorithm runs as follows:

1. Determine $\mathcal{M}_{\Gamma}$ and take, for every $L \in \mathcal{M}_{\Gamma}$, a $\mathfrak{b} \in \mathcal{S}_{\Gamma}$ such that $L=L(\mathfrak{b})$.

2. If there exists a $\mathfrak{b}$ in this list such that $\tau^{\mathfrak{b}}$ is satisfiable, then $\tau$ is satisfiable relative to $\Gamma$. Otherwise $\tau$ is not satisfiable relative to $\Gamma$.

Obviously, all this can be done effectively. The soundness of this algorithm is clear. Completeness is a consequence of the following lemma:

Lemma 24. Suppose $\mathfrak{a}, \mathfrak{b} \in \mathcal{S}_{\Gamma}$. Then

(i) if $L(\mathfrak{a}) \subseteq L(\mathfrak{b})$, then $\tau^{\mathfrak{a}}$ is satisfiable whenever $\tau^{\mathfrak{b}}$ is satisfiable;

(ii) if $L(\mathfrak{a})=L(\mathfrak{b})$, then $\tau^{\mathfrak{a}}$ is satisfiable iff $\tau^{\mathfrak{b}}$ is satisfiable.

Proof. We begin by introducing the notions required for the proof. Suppose that $\tau$ is an $\mathcal{M T}$-term and, as before, $N(\tau)$ is the set of numerical parameters occurring in $\tau$. Suppose also that a frame

$$
\mathfrak{T}=\left\langle W, R,\left(D_{a}^{\leq}\right)_{a \in N(\tau)},\left(D_{a}^{<}\right)_{a \in N(\tau)}\right\rangle
$$

is such that

$$
\left\langle W, R \cup \bigcup_{a \in N(\tau)}\left(D_{a}^{\leq} \cup D_{a}^{<}\right)\right\rangle
$$


is a (possibly infinite) forest of (disjoint) intransitive and irreflexive trees (so here we do not assume that $(q o R)-\left(D^{<} R\right)$ hold). Then a structure of the form

$$
\mathfrak{S}=\left\langle\mathfrak{T}, P_{1}^{\mathfrak{S}}, P_{2}^{\mathfrak{S}}, \ldots\right\rangle,
$$

where $P_{i}^{\mathfrak{S}} \subseteq W$ for $i<\omega$, is called a $\tau$-skeleton. The value $\rho^{\mathfrak{S}}$ of a subterm $\rho$ of $\tau$ in $\mathfrak{S}$ is defined in precisely the same way as for the standard $\mathcal{M T}$-models. The term $\tau$ is satisfied in $\mathfrak{S}$ if $\tau^{\mathfrak{S}} \neq \emptyset$.

The expansion of a $\tau$-skeleton $\mathfrak{S}$ is the $\mathcal{M T}[\tau]$-model

$$
\begin{aligned}
& \mathfrak{S}^{*}=\left\langle\mathfrak{T}^{*}, P_{1}^{\mathfrak{S}}, P_{2}^{\mathfrak{S}}, \ldots\right\rangle, \quad \text { with } \\
& \mathfrak{T}^{*}=\left\langle W, R^{*},\left(E_{a}^{\leq}\right)_{a \in M[\tau]},\left(E_{a}^{<}\right)_{a \in M[\tau]}\right\rangle,
\end{aligned}
$$

where the $\mathfrak{T}^{*}$ is the result of closing $\mathfrak{T}$ under the rules $(q o R)-\left(D^{<} R\right)$ for $M[\tau]$. Say that a $\tau$-skeleton $\mathfrak{S}$ is expandable if, for every subterm $\rho$ of $\tau$, we have

$$
\rho^{\mathfrak{S}}=\rho^{\mathfrak{S}^{*}} \text {. }
$$

LEMma 25. An $\mathcal{M T}$-term $\tau$ is satisfiable iff it is satisfiable in some expandable $\tau$-skeleton.

Proof. The implication $(\Leftarrow)$ follows from definition.

$(\Rightarrow)$ Suppose that $\tau^{\mathfrak{K}} \neq \emptyset$ for some finite $\mathcal{M T}[\tau]$-model

$$
\begin{aligned}
\mathfrak{K} & =\left\langle\mathfrak{F}, P_{1}^{\mathfrak{K}}, P_{2}^{\mathfrak{K}}, \ldots\right\rangle, \quad \text { with } \\
\mathfrak{F} & =\left\langle W, R,\left(D_{a}^{\leq}\right)_{a \in M[\tau]},\left(D_{a}^{<}\right)_{a \in M[\tau]}\right\rangle .
\end{aligned}
$$

Consider the reduction

$$
\mathfrak{F}^{\prime}=\left\langle W, R,\left(D_{a}^{\leq}\right)_{a \in N(\tau)},\left(D_{a}^{<}\right)_{a \in N(\tau)}\right\rangle
$$

of $\mathfrak{F}$ to $N(\tau)$ and apply to it the standard unravelling procedure (see, e.g., [9]). More precisely, take some minimal subset roots $=\left\{w_{1}, \ldots, w_{k}\right\}$ of $W$ such that, for every $w^{\prime} \in W-$ roots, there is a path

$$
w_{i} S_{1} u_{1} S_{2} u_{2} S_{3} \ldots S_{n} u_{n}
$$

where $u_{n}=w^{\prime}$, each $S_{i}$ is one of $R, D_{a}^{\leq}, D_{a}^{<}, a \in N(\tau)$, and $w_{i} \in$ roots. Now construct the frame

$$
\mathfrak{T}=\left\langle\widehat{W}, \widehat{R},\left(\widehat{D}_{a}^{\leq}\right)_{a \in N(\tau)},\left(\widehat{D}_{a}^{<}\right)_{a \in N(\tau)}\right\rangle
$$

where $\widehat{W}$ consists of all finite sequences of the form $\left(w_{i}, u_{1}, \ldots, u_{n}\right)$ for which (37) holds, and for each relation $\widehat{S} \in\left\{\widehat{R}, \widehat{D}_{a}^{\leq}, \widehat{D}_{a}^{<} \mid a \in N(\tau)\right\}$, we have

$$
\left(w_{i}, u_{1}, \ldots, u_{n}\right) \widehat{S}\left(w_{j}, v_{1}, \ldots, v_{m}\right)
$$

iff $m=n+1, i=j, u_{i}=v_{i}$ for $i=1, \ldots, n$, and $u_{n} S v_{m}$ holds in $\mathfrak{F}^{\prime}$. Let

$$
\mathfrak{S}=\left\langle\mathfrak{T}, P_{1}^{\mathfrak{S}}, P_{2}^{\mathfrak{S}}, \ldots\right\rangle
$$

where $\left(w_{i}, u_{1}, \ldots, u_{n}\right) \in P_{j}^{\mathfrak{S}}$ iff $u_{n} \in P_{j}^{\mathfrak{K}}$. It should be clear that $\mathfrak{S}$ is a $\tau$-skeleton such that, for every subterm $\rho$ of $\tau$,

$$
\left(w_{i}, u_{1}, \ldots, u_{n}\right) \in \rho^{\mathfrak{S}} \quad \text { iff } \quad u_{n} \in \rho^{\mathfrak{K}}
$$

Thus, it remains to show that $\mathfrak{S}$ is expandable. 
Let $\mathfrak{S}^{*}$ be the expansion of $\mathfrak{S}$ based on the $\mathcal{M T}$-frame $\mathfrak{T}^{*}$ as defined in (35), (36). We claim that, for every subterm $\rho$ of $\tau$, we have $\rho^{\mathfrak{S}}=\rho^{\mathfrak{S}^{*}}$. The claim is proved by induction on the construction of $\rho$. Here we only show that $\left(\exists^{<a} \rho\right)^{\mathfrak{S}}=\left(\exists^{<a} \rho\right)^{\mathfrak{S}^{*}}$. The inclusion $\subseteq$ is trivial. To prove the converse, suppose $\left(w_{i}, u_{1}, \ldots, u_{n}\right) \in\left(\exists^{<a} \rho\right)^{\mathfrak{S}^{*}}$. Then there is $\left(w_{j}, v_{1}, \ldots, v_{m}\right) \in \rho^{\mathfrak{S}^{*}}$ such that $\left(w_{i}, u_{1}, \ldots, u_{n}\right) E_{a}^{<}\left(w_{j}, v_{1}, \ldots, v_{m}\right)$. Using Lemma $3(\mathrm{e}),(\mathrm{g})$ and the definition of unravelling, it is not hard to see that we then have $u_{n} D_{a}^{<} v_{m}$. By the induction hypothesis, $\left(w_{j}, v_{1}, \ldots, v_{m}\right) \in \rho^{\mathfrak{S}}$, and so, by $(38), v_{m} \in \rho^{\mathfrak{K}}$. Therefore, $u_{n} \in\left(\exists_{a}^{<} \rho\right)^{\mathfrak{K}}$ and, again by $(38),\left(w_{i}, u_{1}, \ldots, u_{n}\right) \in\left(\exists^{<a} \rho\right)^{\mathfrak{S}}$.

Now, clearly, (ii) follows from (i). To prove (i) suppose that $L(\mathfrak{a}) \subseteq L(\mathfrak{b})$ and set $N_{1}=\left\{\mathfrak{a}\left(x_{i}\right) \mid 1 \leq i \leq n\right\}, N_{2}=\left\{\mathfrak{b}\left(x_{i}\right) \mid 1 \leq i \leq n\right\}$. Denote by $M_{1}$ and $M_{2}$ the closure under $(+)$ and $(-)$ of $N_{1}$ and $N_{2}$, respectively, and set $\varepsilon_{1}=\min M_{1}$, $\varepsilon_{2}=\min M_{2}$.

Suppose $\tau^{\mathfrak{b}}$ is satisfiable. By Lemma 25, we can satisfy $\tau^{\mathfrak{b}}$ in an expandable $\tau^{\mathfrak{b}}$-skeleton

$$
\mathfrak{S}_{2}=\left\langle\mathfrak{T}_{2}, P_{1}^{\mathfrak{S}_{2}}, P_{2}^{\mathfrak{S}_{2}}, \ldots\right\rangle
$$

where

$$
\mathfrak{T}_{2}=\left\langle W, R,\left(D_{b}^{\leq}\right)_{b \in N_{2}},\left(D_{b}^{<}\right)_{b \in N_{2}}\right\rangle \text {. }
$$

Denote by $R^{*}, E_{b}^{\leq}, E_{b}^{<}, b \in M_{2}$, the relations in the expansion $\mathfrak{S}_{2}^{*}$ of $\mathfrak{S}_{2}$. Our aim is to construct an $M_{1}$-expandable $\tau^{\mathfrak{a}}$-skeleton satisfying $\tau^{\mathfrak{a}}$.

Let

$$
\mathfrak{S}_{1}=\left\langle\mathfrak{T}_{1}, P_{1}^{\mathfrak{S}_{1}}, P_{2}^{\mathfrak{S}_{1}}, \ldots\right\rangle
$$

where $P_{i}^{\mathfrak{S}_{1}}=P_{i}^{\mathfrak{S}_{2}}$, for $i<\omega$, and

$$
\mathfrak{T}_{1}=\left\langle W, R,\left(F_{a}^{\leq}\right)_{a \in N_{1}},\left(F_{a}^{<}\right)_{a \in N_{1}}\right\rangle
$$

where, for $1 \leq i \leq n$,

$$
F_{\mathfrak{a}\left(x_{i}\right)}^{\leq}=D_{\mathfrak{b}\left(x_{i}\right)}^{\leq}, \quad F_{\mathfrak{a}\left(x_{i}\right)}^{<}=D_{\mathfrak{b}\left(x_{i}\right)}^{<} .
$$

Denote by $R^{*}, G_{a}^{<}, G_{a}^{<}, a \in M_{1}$, the relations in the expansion $\mathfrak{S}_{1}^{*}$ of $\mathfrak{S}_{1}$.

We claim that, for all $u, v \in W$ and $1 \leq i \leq n$,

(1) $G_{\mathfrak{a}\left(x_{i}\right)}^{<} \subseteq E_{\mathfrak{b}\left(x_{i}\right)}^{<}$,

(2) $G_{\mathfrak{a}\left(x_{i}\right)}^{\leq} \subseteq E_{\mathfrak{b}\left(x_{i}\right)}^{\leq}$.

Here we only show (1) and leave (2) to the reader. Let us write $s=u R F v$ if $s$ is a sequence of the form $u=x_{0} S_{0} x_{1} S_{1} \ldots S_{n-1} x_{n}$ such that each $S_{i}$ is one of $R$, $F_{a}^{<}$or $F_{a}^{\leq}, a \in N_{1}$. As before, $a_{s}$ denotes the sum of the parameters that occur in $s$. By $s=u R D v$ we denote sequences that use relations $R, D_{b}^{<}$and $D_{b}^{\leq}$, for $b \in N_{2}$.

Given a sequence $s=u R F v$, denote by $w(s)$ the sequence of the form $u R D v$ that results from $s$ by replacing all $F_{\mathfrak{a}\left(x_{i}\right)}^{<}$and $F_{\mathfrak{a}\left(x_{i}\right)}^{\leq}$with $D_{\mathfrak{b}\left(x_{i}\right)}^{<}$and $D_{\mathfrak{b}\left(x_{i}\right)}^{\leq}$, respectively.

Suppose $(u, v) \in G_{\mathfrak{a}\left(x_{j}\right)}^{<}$. Recall that

$$
\left\langle W, R \cup \bigcup_{a \in N_{1}} F_{a}^{\leq} \cup \bigcup_{a \in N_{1}} F_{a}^{<}\right\rangle
$$


is an intransitive and irreflexive tree. Therefore, the following cases (which do not necessarily exclude each other) are possible:

Case 1. If $u=v$ then $(u, v) \in E_{\mathfrak{b}\left(x_{1}\right)}^{<}$, and the claim follows.

Case 2. There exists $s=u R F v$ such that $a_{s}<\mathfrak{a}\left(x_{j}\right)$. But then we obtain $a_{s}+\varepsilon_{1} \leq \mathfrak{a}\left(x_{j}\right)$. So $w(s)=u R D v$ and, since $L(\mathfrak{a}) \subseteq L(\mathfrak{b})$, we have $a_{w(s)}<\mathfrak{b}\left(x_{j}\right)$. Now, $\varepsilon_{2} \leq \mathfrak{b}\left(x_{j}\right)-a_{w(s)}$, and, therefore, $a_{w(s)}+\varepsilon_{2} \leq \mathfrak{b}\left(x_{j}\right)$. This implies $a_{w(s)}<\mathfrak{b}\left(x_{j}\right)$, and so we derive $(u, v) \in E_{\mathfrak{b}\left(x_{j}\right)}^{<}$.

Case 3. There exists $s=v R F u$ such that $a_{s}<\mathfrak{a}\left(x_{j}\right)$. Then swap $v$ and $u$ and proceed as in Case 2.

Case 4. There exists $s=u R F v$ such that $s$ contains an $F_{a}^{<}$and at least one $F_{a}^{<}$occurs before any $R$ in $s$ and $a_{s} \leq \mathfrak{a}\left(x_{j}\right)$. Then $a_{w(s)} \leq \mathfrak{b}\left(x_{j}\right)$, since $L(\mathfrak{a}) \subseteq L(\mathfrak{b})$, and so $(u, v) \in E_{\mathfrak{b}\left(x_{j}\right)}^{<}$.

Case 5. As in Case 4 but with $u$ and $v$ swapped. Then proceed as in Case 4.

Case 6. There exist $s_{1}=u_{0} R F u, s_{2}=u_{0} R F v$ and $a_{s_{1}}+a_{s_{2}}<\mathfrak{a}\left(x_{j}\right)$. Then $a_{s_{1}}+a_{s_{2}}+\varepsilon_{1} \leq \mathfrak{a}\left(x_{j}\right)$. Again, using $L(\mathfrak{a}) \subseteq L(\mathfrak{b})$, we obtain $a_{w\left(s_{1}\right)}+a_{w\left(s_{2}\right)}+\varepsilon_{2} \leq$ $\mathfrak{b}\left(x_{j}\right)$, and so $(u, v) \in E_{\mathfrak{b}\left(x_{j}\right)}^{<}$.

Case 7. There exist $s_{1}=u_{0} R F u, s_{2}=u_{0} R F v$ such that at least one of $s_{1}, s_{2}$ contains an $F_{a}^{<}$which occurs before any $R$ and $a_{s_{1}}+a_{s_{2}} \leq \mathfrak{a}\left(x_{j}\right)$. Using $L(\mathfrak{a}) \subseteq L(\mathfrak{b})$, we obtain $a_{w\left(s_{1}\right)}+a_{w\left(s_{2}\right)} \leq \mathfrak{b}\left(x_{j}\right)$ and, therefore, $(u, v) \in E_{\mathfrak{b}\left(x_{j}\right)}^{<}$.

This finishes the proof of the claim.

Obviously, for any subterm $\rho$ of $\tau$, we have

$$
\left(\rho^{\mathfrak{a}}\right)^{\mathfrak{S}_{1}}=\left(\rho^{\mathfrak{b}}\right)^{\mathfrak{S}_{2}} .
$$

It remains to show by induction that, for any subterm $\rho$ of $\tau$,

$$
\left(\rho^{\mathfrak{a}}\right)^{\mathfrak{S}_{1}^{*}}=\left(\rho^{\mathfrak{b}}\right)^{\mathfrak{S}_{2}^{*}}
$$

The interesting steps are $\rho=\forall^{<x_{i}} \rho_{0}$ and $\rho=\forall \leq x_{i} \rho_{0}$. We only consider the former.

Suppose $u \notin\left(\rho^{\mathfrak{a}}\right)^{\mathfrak{S}_{1}^{*}}$. Then there is $v$ such that $(u, v) \in G_{\mathfrak{a}\left(x_{i}\right)}^{<}$and $v \notin\left(\rho_{0}^{\mathfrak{a}}\right)^{\mathfrak{S}_{1}^{*}}$. By the induction hypothesis, $v \notin\left(\rho_{0}^{\mathfrak{b}}\right)^{\mathfrak{S}_{2}^{*}}$. By the claim above, $(u, v) \in E_{\mathfrak{b}\left(x_{i}\right)}^{<}$. Therefore, $u \notin\left(\rho^{\mathfrak{b}}\right)^{\mathfrak{S}_{2}^{*}}$.

Suppose $u \notin\left(\rho^{\mathfrak{b}}\right)^{\mathfrak{S}_{2}^{*}}$. It follows that $u \notin\left(\rho^{\mathfrak{b}}\right)^{\mathfrak{S}_{2}}$, and so $u \notin\left(\rho^{\mathfrak{a}}\right)^{\mathfrak{S}_{1}}$. But then, by the induction hypothesis, we obtain $u \notin\left(\rho^{\mathfrak{a}}\right)^{\mathfrak{S}_{1}^{*}}$.

In general, it is not known whether there is an elementary upper bound for the complexity of this procedure. However, in some cases it can be considerably simplified:

Corollary 26. (i) Suppose that $\Gamma$ consists of 'pure' strict inequalities

$$
\left\{0<x_{1}, x_{1}<x_{2}, x_{2}<x_{3}, \ldots, x_{n-1}<x_{n}\right\}
$$

and $\tau$ is an $\mathcal{M T}[V]$-term with variables $\left\{x_{1}, \ldots, x_{n}\right\}$. Then $\tau$ is satisfiable relative to $\Gamma$ iff $\tau^{\mathfrak{a}}$ is satisfiable, where $\mathfrak{a}\left(x_{1}\right)=1$ and, for $1<i \leq n, \mathfrak{a}\left(x_{i}\right)=$ $1+\frac{1}{n+2-i}$.

(ii) Suppose that $\Gamma$ consists of inequalities

$$
\left\{x_{2} \geq a_{1} x_{1}, x_{3} \geq a_{2} x_{2}, \ldots, x_{n} \geq a_{n-1} x_{n-1}\right\},
$$


where the $a_{i}>1$ are natural numbers, and $\tau$ is an $\mathcal{M T}[V]$-term with variables $\left\{x_{1}, \ldots, x_{n}\right\}$. Then $\tau$ is satisfiable relative to $\Gamma$ iff $\tau^{\mathfrak{a}}$ is satisfiable, where $\mathfrak{a}\left(x_{1}\right)=1$ and, inductively, $\mathfrak{a}\left(x_{i+1}\right)=a_{i} \cdot \mathfrak{a}\left(x_{i}\right)$.

In both cases the satisfiability problem for $\tau$ relative to $\Gamma$ is EXPTIME-complete.

Proof. In both cases the set $\mathcal{M}_{\Gamma}$ contains just one set of inequalities $L$ and $L(\mathfrak{a})=L$.

§6. Conclusion. In this paper we have defined and investigated a new framework for integrating qualitative and quantitative aspects of reasoning about metric spaces and their induced topologies. A number of interesting open problems arise within this framework if we (i) consider satisfiability of $\mathcal{M T}$-formulas in various important classes of metric spaces and/or (ii) weaken or strengthen the expressive power of $\mathcal{M T}$.

- For example, it would be of interest to investigate the logic determined by metric spaces whose induced topological spaces are connected. We conjecture that this logic can be axiomatised by adding the connectivity axiom (2) to MT and that results similar to those for MT can be obtained.

- We have proved that $L(\mathbb{R})$ is decidable. Does there exist a transparent axiomatisation of this logic? What is the computational complexity of $L(\mathbb{R})$ ?

- Although the logic of $\mathbb{R}^{2}$ (and its subspaces) is undecidable, we do not know whether it is recursively enumerable. Nor is it known what happens if we omit the operators $\exists \leq a$.

- What is the computational complexity of satisfiability of $\mathcal{M T}[V]$-terms in metric spaces?

- In [26], we have investigated the (non-topological) metric language with operators $\exists^{\leq a}$ and $\exists^{>a}$ ('somewhere outside the closed sphere of radius $a^{\prime}$ ) and proved the decidability of satisfiability in arbitrary metric spaces. Is the satisfiability problem for the extension of this language with topological (interior and closure) operators decidable as well?

- The addition of nominals (atomic terms interpreted as singleton sets) would make the language powerful enough for reasoning about concepts, similarities, and prototypes in combinations with, e.g., description logics [18, 28]. It is an interesting open problem whether the resulting language is still decidable. Note that the nominals would require new axioms like

$$
\diamond\{n\}=\{n\}, \quad \diamond \exists^{<a}\{n\} \sqsubseteq \exists^{\leq a}\{n\},
$$

where $n$ is a nominal.

Acknowledgements. The work on this paper was partially supported by the U.K. EPSRC research grants GR/S61966/01 and GR/S61973/01. Thanks are due to Ivan Zakharyaschev for his help and comments. We are also grateful to the anonymous referee whose remarks and suggestions helped us to improve the paper. 
REFERENCES

[1] M. Aiello, J. van Benthem, and G. Bezhanishvili, Reasoning about space: the modal way, Journal of Logic and Computation, vol. 6 (2003), pp. 889-920.

[2] P. S. Alexandroff, Diskrete Räume, Matematicheskii Sbornik, vol. 2 (44) (1937), pp. 501-518.

[3] R. Alur, T. Feder, and T. Henzinger, The benefits of relaxing punctuality, Journal of the ACM, vol. 43 (1996), pp. 116-146.

[4] R. Alur and T.A. Henzinger, A really temporal logic, Journal of the ACM, vol. 41 (1994), pp. 181-204.

[5] C. Areces, P. Blackburn, and M. Marx, The computational complexity of hybrid temporal logics, Logic Journal of the IGPL, vol. 8 (2000), pp. 653-679.

[6] S. Artemov, J. Davoren, and A. Nerode, Modal logics and topological semantics for hybrid systems, Technical Report MSI 97-05, Cornell University, 1997.

[7] B. Bennett, Modal logics for qualitative spatial reasoning, Logic Journal of the IGPL, vol. 4 (1996), pp. 23-45.

[8] G. Bezhanishvili and M. Gehrke, A new proof of completeness of S4 with respect to the real line, 2002, Preprint PP-2002-06 of ILLC, University of Amsterdam.

[9] P. Blackburn, M. de Rijke, and Y. Venema, Modal logic, Cambridge University Press, 2001.

[10] E. Börger, E. Grädel, and Yu. Gurevich, The classical decision problem, Perspectives in Mathematical Logic, Springer, 1997.

[11] N. Bourbaki, General topology, part 1, Hermann, Paris and Addison-Wesley, 1966.

[12] A. Chagrov and M. Zakharyaschev, Modal logic, Oxford Logic Guides, vol. 35, Clarendon Press, Oxford, 1997.

[13] P. Clote and R. Backofen, Computational molecular biology, John Wiley \& Sons, 2000 .

[14] A. Cohn and S. HAZARIKA, Qualitative spatial representation and reasoning: an overview, Fundamenta Informaticae, vol. 46 (2001), pp. 1-29.

[15] D. Dubois, H. Prade, F. Esteva, P. Garcia, and L. Godo, A logical approach to interpolation based on similarity relations, International Journal of Approximate Reasoning, vol. 17 (1997), pp. 1-36.

[16] M. Egenhofer and R. Franzosa, Point-set topological spatial relations, International Journal of Geographical Information Systems, vol. 5 (1991), pp. 161-174.

[17] F. Esteva, P. Garcia, L. Godo, and R. Rodriguez, A modal account of similaritybased reasoning, International Journal of Approximate Reasoning, vol. 16 (1997), pp. 235260.

[18] P. Gärdenfors, Conceptual spaces, The MIT Press, 2000.

[19] P. GäRDENFORS and M.A. Williams, Reasoning about categories in conceptual spaces, Proceedings of the 7th international joint conference on artificial intelligence (ijcai), Morgan Kaufmann, 2001, pp. 385-392.

[20] R. Goldblatt, Mathematics of modality, CSLI Lecture Notes, no. 43, CSLI Publications, Stanford, 1993.

[21] M. Grigni, D. Papadias, and Ch. Papadimitriou, Topological inference, Proceedings of the 14th international joint conference on artificial intelligence (ijcai), Morgan Kaufmann, 1995, pp. 901-906.

[22] D. Harel, D. Kozen, and J. Tiuryn, Dynamic logic, MiT Press, 2000.

[23] Y. Hirshfeld and A. Rabinovich, Quantitative temporal logic, Proceedings of computer science logic 1999, Springer, 1999, pp. 172-187.

[24] D. Huttenlocher, G. Klanderman, and W. Rucklidge, Comparing images using the Hausdorff distance, IEEE Transactions on Pattern Analysis and Machine Intelligence, vol. 15 (1993), pp. 850-863.

[25] P. Kremer, G. Mints, and V. Rybakov, Axiomatizing the next-interior fragment of dynamic topological logic, The Bulletin of Symbolic Logic, vol. 3 (1997), pp. 376-377.

[26] O. Kutz, H. Sturm, N.-Y. Suzuki, F. Wolter, and M. Zakharyaschev, Logics of metric spaces, ACM Transactions on Computational Logic, vol. 4 (2003), pp. 260-294. 
[27] R. LADNER, The computational complexity of provability in systems of modal logic, SIAM Journal on Computing, vol. 6 (1977), pp. 467-480.

[28] C. Lutz, F. Wolter, and M. Zakharyaschev, A tableau algorithm for reasoning about concepts and similarity, Automated reasoning with analytic tableaux and related methods (M. C. Mayer and F. Pirri, editors), LNCS, vol. 2796, Springer, 2003.

[29] J.C.C. MCKinsey and A. TARski, The algebra of topology, Annals of Mathematics, vol. 45 (1944), pp. 141-191.

[30] G. MinTs, A completeness proof for propositional $\mathbf{S 4}$ in Cantor space, Logic at work (E. Orlowska, editor), Springer, 1999, pp. 79-88.

[31] J. REnZ and B. NeBel, On the complexity of qualitative spatial reasoning, Artificial Intelligence, vol. 108 (1999), pp. 69-123.

[32] V. Shehtman, "Everywhere" and "Here", Journal of Applied Non-classical logic, vol. 9 (1999), pp. 369-379.

[33] E. SpaAn, Complexity of modal logics, Ph.D. thesis, Department of Mathematics and Computer Science, University of Amsterdam, 1993.

[34] A. TARski, Der Aussagenkalkül und die Topologie, Fundamenta Mathematicae, vol. 31 (1938), pp. 103-134.

[35] F. Wolter and M. Zakharyaschev, Reasoning about distances, Proceedings of the 18th international joint conference on artificial intelligence (IJCAI 2003), Morgan Kaufmann, 2003, pp. 1275-1280.

[36] M. Worboys, GIS: A computational perspective, Taylor \& Francis, 1995.

DEPARTMENT OF COMPUTER SCIENCE

UNIVERSITY OF LIVERPOOL

LIVERPOOL L69 7ZF, U.K.

E-mail: frank@csc.liv.ac.uk

$U R L:$ http://www.csc.liv.ac.uk/ ${ }^{\sim}$ rank

DEPARTMENT OF COMPUTER SCIENCE

KING'S COLLEGE LONDON

STRAND, LONDON WC2R 2LS, U.K.

E-mail:mz@dcs.kcl.ac.uk

URL: http://www.dcs.kcl.ac.uk/staff/mz 